\title{
Order-Isomorphisms between Cones of Continuous Functions $\left(^{(*)}\left(^{* *}\right)\right.$.
}

\author{
JUAN Jorge SchäfFer (Pittsburgh, Pennsylvania)
}

Summary. - This paper studies the strueture of order-isomorphisms between the cones of strictlypositive-valued elements of subspaces of two spaces of continuous functions; a complete description is obtained in some cases. Conditions are also found that ensure that such an orderisomorphism is linear provided it is homogeneous along at least one ray.

\section{1. - Introduction.}

This paper continues the study of the structure of mappings between cones begun in [6] and [7]. Here we are specifically concerned with order-isomorphisms between the cones of strictly positive elements of quite arbitrary spaces consisting of continuous functions on compact spaces. Our intention is twofold: first, to obtain as explicit a description of the structure of such mappings as we can; second, to find conditions under which such mappings must be linear. Consideration of these cones of continuous functions is motivated by two facts: they are the most obvious concrete examples of genuine faceless cones (in the language of [7]); and they serve to represent all abstract genuine faceless cones up to linear isomorphisms (see [8]).

When the spaces in question are those of all continuous functions on the respective domains, Kaplansky's Theorem ([5]) implies that, if there exists a bijection between the strictly positive cones that, together with its inverse, preserves the value-wise order (a " $\leqslant-\leqslant$-isomorphism", which in this case is just a lattice-isomorphism), then the domains are homeomorphic. We obtain a complete characterization of such bijections that, together with their inverses, preserve the value-wise strict-order ("<-<-isomorphisms $")$ : such a mapping acts by transplanting each function by means of a homeomorphism between the domains, followed by a suitable distortion of the scale of values at each point (Theorem 6.5). This not unexpected-and probably known-result remains valid for other spaces of continuous functions, in particular for sublattices of the spaces of all continuous functions (remarks following Theorem 6.1, Examples 6.3, Proposition 6.4), and serves as a prototype for a partial representation of such mappings in more general cases (Theorem 6.1, results in Section 7).

(*) Entrata in Redazione il 12 ottobre 1977.

$\left(^{* *}\right)$ This work was supported in part by National Seience Foundation Grant MCS75-06667. 
The method for obtaining these structural theorems is a pale order-theoretical imitation of the usual argument employed in proving the Banach-Stone Theorem on linear isometries between spaces of continuous functions (and hence is a distant relative of the method of proof of Kaplansky's Theorem as well). There, the maximal vector-lattice ideals are naturally associated with the individual points of the domain; the order-theoretical analogues, maximal directed sets of non-negative-valued functions (Section 3), are more complicated: for a given space of continuous functions, two points of the domain may well be separated by an element of the space that vanishes at one and not the other, but not by a non-negative-valued one.

In [7] certain conditions on the abstract cones there considered (e.g. rotundity) ensured that every isomorphism between them is necessarily linear ([7; Theorem A, Corollary A1]); results of this type go back, in a way, to the work of ALEKSANDRov and OvơnnNIKova [1]. Here, on the contrary, we are interested in weak conditions on the order-isomorphism that will ensure its linearity. Specifically, we investigate the consequences of the assumption that the order-isomorphism is homogeneous along one ray (Theorem 8.1), and find that in many cases this is enough to force it to be linear (Corollaries 8.2, 8.3, 8.4, Examples 8.5, Theorem 8.6). These are mostly cases to which [7; Theorem B] is not applicable. These results can, in turn, be applied to order-isomorphisms between abstract cones: in [8] it is shown, using Theorem 8.6, that homogeneous order-isomorphisms between any genuine faceless cones are necessarily linear.

We mention in passing that, although in this paper we only examine order-isomorphisms between (open) positive cones, the results can be immediately applied to similar mappings between more general "upward-saturated "subsets of the spaces in question, and in particular between the whole spaces. For instance, it is an immediate consequence of Corollary 8.4 that every order-isomorphism between the spaces of all continuous functions on two compact Hausdorff spaces is necessarily linear if-to cite a very special case-it maps each constant into the constant with the same value.

The author is grateful to Professors I. Namroka, W. Nold, and R. R. Phelps for helpful discussion of several aspects of this work.

We mention some items of terminology and notation. We use $:=$ or $: \Leftrightarrow$ to indieate an equality or a logical equivalence, respectively, in which the left-hand side is defined by the right-hand side.

If $S$ is a set, $\mathfrak{F}(S)$ denotes the power-set of $S$. Let $\varphi: A \rightarrow B$ be a mapping, $S$ a subset of $A$, and $T$ a subset of $B$. We write $\varphi_{>}(S):=\{\varphi(x) \mid x \in S\}$ for the image of $S$ under $\varphi$ and $\varphi^{<}(T):=\{x \in B \mid \varphi(x) \in T\}$ for the pre-image of $T$ under $\varphi$. In particular, Rng $\varphi:=\varphi_{>}(A)$ is the range of $\varphi$. If $\varphi_{>}(S) \subset T$, the restriction $\left.\varphi\right|_{S} ^{T}: S \rightarrow T$ of $\varphi$ is defined by $\left(\left.\varphi\right|_{S} ^{T}\right)(x):=\varphi(x)$ for all $x \in S$; in particular, $\left.\varphi\right|_{S}:=\left.\varphi\right|_{S} ^{B}$.

Let $L$ and $L^{\prime}$ be sets and $\varrho$ and $\varrho^{\prime}$ relations in $L$ and $L^{\prime}$, respectively. A mapping $\varphi: L \rightarrow L^{\prime}$ is a $\varrho^{-} \varrho^{\prime}$-isomorphism if $\varphi$ is bijective and $x \varrho y \Leftrightarrow \varphi(x) \varrho^{\prime} \varphi(y)$ for all $x, y \in L$.

If $L, \triangleleft$ is an ordered set, a $\triangleleft$-direeted subset of $L$ is a subset $D$ of $L$ such that for all $x, y \in D$ there exists $z \in D$ such that $x \triangleleft z$ and $y \triangleleft z$. A maximal $\triangleleft$-directed subset 
of $L$ is a maximal element, with respect to inclusion, of the collection of all $\triangleleft$-directed subsets of $L$.

The symbol $\boldsymbol{R}$ denotes the set of all real numbers, and we set $\boldsymbol{P}:=\{s \in \boldsymbol{R} \mid s \geqslant 0\}$ and $\boldsymbol{P} \times:=\boldsymbol{P} \backslash\{0\}$.

Let $V$ be a (real) linear space. When $w \in V$ and $A, B \subset V$ we use such notations as $A+B:=\{u+v \mid u \in A, v \in B\}, \quad w-A:=\{w-u \mid u \in A\}, \quad \boldsymbol{P}^{\times} w:=\left\{\alpha w \mid \alpha \in \boldsymbol{P}^{\times}\right\}$. The linear span of a subset $A$ of $V$ is denoted by $\operatorname{Sp} A$.

A subset $C$ of a real linear space is a (linear) cone if it is stable under addition and under multiplication by strictly positive numbers, i.e., if $C+C \subset C$ and $\boldsymbol{P} \times O \subset C$. If $O$ is a non-empty cone, its linear span is $\operatorname{sp} C=O-O$.

Let $C$ and $C^{\prime}$ be cones. We say that a mapping $\lambda: C \rightarrow C^{\prime}$ is linear if it is the restriction of a linear mapping from $\mathrm{Sp} O$ to $\mathrm{Sp} C^{\prime}$; this is the case if and only if $\lambda$ preserves addition and multiplication by strictly positive numbers. If $\lambda$ is linear and bijective, then $\lambda^{-1}$ is also linear and hence $\lambda$ is a cone-isomorphism.

\section{2. - Positive cones and orders.}

Let $E$ be a non-empty compact Hausdorff space, and Cont $(E)$ the linear space of all continuous real-valued functions on $E$. On Cont $(E)$, as well as on each of its subsets, we consider the value-wise order $\leqslant$ and the value-wise strict-order $<$ defined by

$$
\begin{aligned}
& f \leqslant g: \Leftrightarrow(\forall p \in E, f(p) \leqslant g(p)) \\
& f<g: \Leftrightarrow(\forall p \in E, f(p)<g(p))
\end{aligned}
$$

for all $f, g \in \operatorname{Cont}(E)$. We define the cones $\operatorname{Cont}^{+}(E):=\{f \in \operatorname{Cont}(E) \mid 0<f\}$ and $\overline{\text { Cont }}^{+}(E):=\{f \in \operatorname{Cont}(E) \mid 0 \leqslant f\}$.

Let $W$ be a (linear) subspace of $\operatorname{Cont}(E)$ that satisfies

$\left(W_{1}\right):$

$$
W^{+}:=W \cap \operatorname{Cont}^{+}(E) \neq \emptyset \text {. }
$$

$W^{+}$is a cone, and it follows from $\left(W_{1}\right)$ that

$$
W=\operatorname{sp} W^{+}=W^{+}-W^{+}
$$

indeed, choose $w \in W^{+}$; let $f \in W$ be given, and choose $\alpha \in \boldsymbol{P}^{\times}$such that $\alpha>\max (f / w)$; then $\alpha w, \alpha w-f \in W^{+}$and $f=\alpha w-(\alpha w-f)$.

We set $\bar{W}^{+}:=W \cap \overline{\operatorname{Cont}}^{+}(E)$, and observe that $\bar{W}^{+}=\left\{f \in W \mid f+W^{+} \subset W^{+}\right\}$ and that

$$
\left\{\begin{array}{l}
f<g \Leftrightarrow g \in f+W^{+} \\
f \leqslant g \Leftrightarrow g \in f+\bar{W}^{+} \Leftrightarrow g+W^{+} c f+W^{+}
\end{array}\right.
$$


for all $f, g \in W$. We note in particular that

$$
f \leqslant g \Leftrightarrow\left(\forall h \in W^{+}, g<h \Rightarrow f<h\right) \quad \text { for all } f, g \in W^{+}
$$

the same statement holds, of course, with $W^{+}$replaced in both places by $W$. Thus $\leqslant$ is determined by $<$ in a purely order-theoretical manner.

In the language of [7], $W^{+}$is a "genuine faceless cone ", and $\bar{W}+$ is its "closure" ([7; Proposition 2.4]). The corresponding "open-order " and "closed-order" on $W$ are the restrictions of $<$ and $\leqslant$, respectively, to $W$. We shall not use this terminology in the present paper, however, although this connection with [7] supplies much of our motivation.

Later we shall impose on $W$, in addition to $\left(W_{1}\right)$, the following "separation condition »:

$\left(W_{2}\right):$ For all $p, q \in E$ with $p \neq q$, there exists $w \in W$ such that $w(p)=0 \neq w(q)$.

It is easy to see that, on account of $\left(\mathrm{W}_{1}\right)$, Condition $\left(\mathrm{W}_{2}\right)$ is equivalent to: For all $p, q \in E$ with $p \neq q$ and all $\alpha, \beta \in \boldsymbol{R}$ there exists $w \in W$ with $w(p)=\alpha, w(q)=\beta$.

In a certain sense, imposing Condition $\left(\mathrm{W}_{2}\right)$ is not very restrictive for our purposes, in view of the following simple result. We shall not, however, pursue the question of using this proposition to reformulate our results withont Condition $\left(W_{2}\right)$.

2.1. Proposition. - Let $E$ be a non-empty compact Hausdorff space, and $W$ a subspace of Cont $(E)$ satisfying $\left(W_{1}\right)$. Choose $w \in W^{+}$. Then there exist a topological quotient mapping $\omega: E \rightarrow E_{0}$, where $E_{0}$ is a (non-empty compact) Hausdorff space, and a subspace $W_{0}$ of cont $\left(E_{3}\right)$ satisfying $\left(\mathrm{W}_{1}\right)$ and $\left(\mathrm{W}_{\mathrm{z}}\right)$ such that the mapping $f \mapsto w(f \circ \omega)$ is a linear isomorphism from $W_{0}$ to $W$, and hence has a restriction to a coneisomorphism from $W_{0}^{+}$to $W^{+}$.

Proof. - Define the equivalence relation $\sim$ on $E$ by

$$
p \sim q: \Leftrightarrow(\forall f \in W, f(p) / w(p)=f(q) / w(q))
$$

for all $p, q \in E$, and choose a topological quotient mapping $\omega: E \rightarrow E_{0}$ with respect to this equivalence relation. Since the elements of $W$ are continuous functions, the graph of $\sim$ is closed, and hence $E_{0}$ is a Hausdorff space. Set $W_{0}:=\left\{f \in \operatorname{Cont}\left(E_{0}\right) \mid\right.$ $w(f \circ \omega) \in W\}$. The remainder of the proof is a straiforward verification.

We mention several examples of spaces satisfying Conditions $\left(W_{1}\right)$ and $\left(W_{2}\right)$.

2.2. ExaMples. - (a) For any non-empty compact Hausdorff space $E$, $W:=$ Cont $(E)$ is itself of course an instance of a subspace of Cont $(E)$ satisfying $\left(\mathrm{W}_{1}\right)$ and $\left(\mathrm{W}_{2}\right)$. In addition, Cont $(E)$ is lattice-ordered by the order $\leqslant$, the lattice operations being the valuewise infimum and supremum; we reserve the notations inf and sup (without subscripts) to denote them. 
(b) Let $X$ be a locally convex Hausdorff topological linear space, and $E$ a compact convex subset of $X$. Let Aff $(E)$ be the space consisting of the restrictions to $E$ of all continuous real-valued affine functions on $X$. Then the subspace $W:=\operatorname{Aff}(E)$ of Cont $(E)$ satisfies $\left(W_{1}\right)$, since it contains all strictly positive constants, and satisfies $\left(W_{2}\right)$ by virtue of the Hahn-Banach Theorem. We write $\overline{\operatorname{Aff}}^{+}(E):=W^{+}$, $\overline{\mathrm{Aff}}^{+}(E):=\bar{W}^{+}$.

(c) Let $X$ be a finite-dimensional real affine space (with its natural topology), and $E$ a compact subset of $X$ with non-empty interior. Let Anal $(E)$ be the subspace of Cont $(E)$ consisting of all elements of Cont $(E)$ that are real-analytic in the interior of $E$. Then $W:=\operatorname{Anal}(E)$ satisfies $\left(W_{1}\right)$ and $\left(W_{2}\right)$. We write $\operatorname{Anal}^{+}(E):=W^{+}$, $\overline{\mathrm{Anal}}^{+}(E):=\bar{W}^{+}$.

(d) With $X$ and $E$ as in (c), let $\mathrm{Pol}(E)$ be the space consisting of the restrictions to $E$ of all real-valued polynomial functions on $X$ (note that the restriction operation is injective). Then $W:=\operatorname{Pol}(E)$ is a subspace of cont $(E)$ satisfying $\left(W_{1}\right)$ and $\left(W_{2}\right)$ We write $\mathrm{Pol}^{+}(E):=W^{+}, \overline{\mathrm{Pol}}^{+}(E):=\bar{W}^{+}$.

\section{3. - The collection $\mathfrak{Z}(W)$ and maximal directed subsets.}

Throughout this section, we shall assume that $E$ is a non-empty compact Hausdorff space and that $W$ is a subspace of Cont $(E)$ satisfying $\left(W_{1}\right)$. Our goal is to characterize the maximal $\leqslant$-directed subsets of $\bar{W}^{+} \backslash W^{+}$.

We define the collection $3(W)$ of subsets of $E$ as the set of all $Z \in \Re(E)$ that satisfy the following three conditions:

$\left(Z_{0}\right) \quad Z \neq \emptyset$

$\left(\mathrm{Z}_{1}\right)$ for every $p \in Z$ and $f \in \bar{W}^{+}, f(p)=0$ implies $f_{>}(Z)=\{0\}$;

$\left(Z_{2}\right)$ for every $q \in E \backslash Z$ there exists $f \in \bar{W}^{+}$such that $f_{>}(Z)=\{0\}$ and $f(q)>0$.

We are particularly interested in those elements of $3(W)$ that are singletons, and define $P(W):=\{p \in E \mid\{p\} \in \mathcal{Z}(W)\}$, noting that

$$
P(W)=\left\{p \in E \mid \forall q \in E \backslash\{p\}, \exists j \in \bar{W}^{+}, f(p)=0<f(q)\right\} .
$$

3.1. Propostition. - $3(W)$ is a disjoint collection of compact sets.

Proof. - Let $Z \in \mathcal{Z}(W)$ be given; by $\left(Z_{0}\right)$ we may choose $p \in Z$. By $\left(\mathbf{Z}_{1}\right)$ and $\left(\mathbf{Z}_{2}\right)$,

$$
Z=\bigcap\left\{f^{<}(\{0\}) \mid f \in \bar{W}^{+}, f(p)=0\right\}
$$

hence $Z$ is closed, and therefore compact. If $Z_{1}, Z_{2} \in 3(W)$ and $p \in Z_{1} \cap Z_{2},(3.2)$ shows that $Z_{1}=Z_{2}$. Thus $\mathfrak{Z}(W)$ is a disjoint collection. 
We define the mapping $J_{W}: \mathfrak{F}(E) \rightarrow \mathfrak{P}\left(\bar{W}^{+}\right)$by

$$
J_{W}(A):=\left\{f \in \bar{W}^{+} \mid f_{>}(A)=\{0\}\right\} \quad \text { for all } A \in \mathfrak{P}(E) \text {. }
$$

We are now able to state the characterization we were looking for.

3.2. Proposition. - The mapping $J_{W}$ has a restriction to a bijection from $3(W)$ to the collection of all maximal $\leqslant$-directed subsests of $\overline{W^{+}} \backslash W^{+}$.

The proof of this proposition depends on three lemmas.

3.3. Lemna. - For every $A \in \mathfrak{F}(E), J_{W}(A)$ is a $\leqslant$-directed subset of $\bar{W}^{+} \backslash W^{+}$.

Proof. $-J_{W}(\emptyset)=\emptyset$ is indeed $a \leqslant$-directed subset of $\bar{W}^{+} \backslash W^{+}$. Let $A \in \mathfrak{B}(E) \backslash\{\emptyset\}$ be given. Clearly, $J_{W}(A) \subset \bar{W}^{+} \backslash W^{+}$. If $f, g \in J_{W}(A)$, then $f+g \in J_{W}(A)$ and $f \leqslant f+g$ and $g \leqslant f+g$. Thus $J_{W}(A)$ is $\leqslant$-directed.

3.4. LempaA. - Every $\leqslant$-directed subset of $\overline{W^{+} \backslash} \backslash W^{+}$is included in $J_{W}(\{p\})$ for some $p \in E$.

Proof. - Let $D$ be a given $\leqslant$-directed subset of $\bar{W}^{+} \backslash W^{+}$. Then $\left\{f^{<}(\{0\}) \mid f \in D\right\}$ is a J-directed collection of non-empty closed subsets of $E$. Since $E$ is a non-empty compact space, the intersection of the collection is not empty. Then $D \subset J_{W}(\{p\})$ for any choice of $p$ in this intersection.

3.5. LemMa. - Let $Z \in \mathfrak{Z}(W)$ and $A \in \mathfrak{P}(E)$ be given. The following statements are equivalent:

(i) $J_{W}(Z)=J_{W}(A)$;

(ii) $J_{W}(Z) \subset J_{W}(A)$ :

(iii) $\emptyset \neq A \subset \dot{Z}$.

Proof. - (i) implies (ii). This is trivial.

(ii) implies (iii). If $A=\emptyset$, then $J_{W}(Z) \neq \emptyset$ is not included in $J_{W}(A)=\emptyset$. Suppose $A \backslash Z \neq \emptyset$, and choose $q \in A \backslash Z$. By $\left(Z_{2}\right)$ we may choose $f \in \bar{W}^{+}$such that $f_{>}(Z)=$ $=\{0\}, f(q)>0$. Thus $f \in J_{W}(Z) \backslash J_{W}(A)$, so that (ii) fails to hold.

(iii) implies (i). Suppose that $\emptyset \neq A \subset Z$. It follows trivially from the definition that $J_{W}(Z) \subset J_{W}(A)$. Choose $p \in A \subset Z$. For every $f \in J_{W}(A)$ we have $f(p)=0$; by $\left(Z_{1}\right), f_{>}(Z)=\{0\}$, so that $f \in J_{W}(Z)$. Thus $J_{W}(A) \subset J_{W}(Z)$.

Proof of Proposition 3.2. - If $Z_{1}, Z_{2} \in \tilde{\zeta}(W)$ and $J_{W(}\left(Z_{1}\right)=J_{W}\left(Z_{2}\right)$, it follows by Lemma 3.5 that $Z_{1}=Z_{2}$. The restriction of $J_{W}$ to $Z(W)$ is therefore injective.

Let $Z \in \mathfrak{Z}(W)$ be given. By Lemma $3.3, J_{W}(Z)$ is a $\leqslant$-directed subset of $\bar{W}^{+} \backslash W^{+}$. Let $D$ be a $\leqslant$-directed subset of $\bar{W}^{+} \backslash W^{+}$such that $J_{W}(Z) \subset D$. By Lemma 3.4, we 
may choose $p \in E$ such that $J_{W}(Z) \subset D \subset J_{W}(\{p\})$. By Lemma 3.5, equality must hold throughout. We conclude that $J_{W}(Z)$ is a maximal $\leqslant$-directed subset of $\overline{W^{+}} \backslash W^{+}$.

Conversely, let $D$ be a maximal $\leqslant$-directed subset of $\bar{W}^{+} \backslash W^{+}$, and define $Z:=\bigcap\left\{f^{<}(\{0\}) \mid f \in D\right\}$. Then $D \subset J_{W}(Z)$, and $J_{W}(Z)$ is a $\leqslant$-directed subset of $\bar{W}+\backslash W^{+}$ by Lemma 3.3. Therefore $D=J_{W}(Z)$. To complete the proof it remains to show that $Z \in \mathfrak{Z}(W)$.

By Lemma 3.4, $Z$ satisfies $\left(Z_{0}\right)$; it satisfies $\left(Z_{2}\right)$ by its very definition. We show that it also satisfies $\left(Z_{1}\right)$. Let $p \in Z$ and $f \in \bar{W}^{+}$be given, with $f(p)=0$. By Lemma 3.3, $J_{W}\left(Z \cap f^{<}(\{0\})\right)$ is a $\leqslant$-directed subset of $\bar{W}^{+} \backslash W^{+}$, and $D \subset J_{W}(Z) \subset J_{W}\left(Z \cap f^{<}(\{0\})\right)$. On account of the maximality of $D$, equality must hold; therefore $f \in J_{W}\left(Z \cap f^{<}(\{0\})\right)=$ $=J_{W}(Z)$, so that $f_{>}(Z)=\{0\}$. This shows that $Z$ satisfies $\left(Z_{1}\right)$.

3.6. Proposition. - Let $Z \in \mathfrak{Z}(W)$ and $f, g \in W$ be given. Then $\left.f\right|_{z}<\left.g\right|_{z}$ if and only if $f<g+h$ for some $h \in J_{W}(Z)$

The proof of this proposition depends on the following lemma.

3.7. LemMa. - If $Z \in \mathcal{Z}(W)$ and $A$ is a slosed subset of $E$ that is disjoint from $Z$, there exists $k \in J_{W}(Z)$ such that $\left.k\right|_{A}>0$.

Proof. - Since $J_{W}(Z)$ is a $\leqslant$-directed set, by Lemma 3.3, we see that $\left\{A \cap f^{<}(\{0\}) \mid f \in\right.$ $\left.\in J_{W}(Z)\right\}$ is a $\supset$-directed collection of closed subsets of $E$. By $\left(Z_{2}\right)$, its intersection is $A \cap Z=\emptyset$. Since $E$ is a compact space, we may choose $k \in J_{W}(Z)$ such that $A \cap$ $\cap k^{<}(\{0\})=\emptyset$; this $k$ satisfies the condition of the statement.

Proof of Proposition 3.6. - If $h \in J_{W}(Z)$ and $f<g+h$, we have $\left.f\right|_{z}<\left.(g+h)\right|_{z}=$ $=\left.g\right|_{z}$. We now assume, conversely, that $\left.f\right|_{z}<\left.g\right|_{z}$, and set $A:=\{q \in E \mid g(q) \leqslant f(q)\}$. Then $A$ is a closed subset of $E$ and is disjoint from $Z$. If $A=\emptyset$, we set $h:=0 \in J_{W}(Z)$ and find $f<g=g+h$. Assume now that $A \neq \emptyset$; by Lemma 3.7, we may choose $k \in J_{W}(Z)$ such that $k_{A}>0$. We set

$$
h:=\left(1+\max \frac{\left.(f-g)\right|_{A}}{\left.k\right|_{A}}\right) k \in J_{W}(Z)
$$

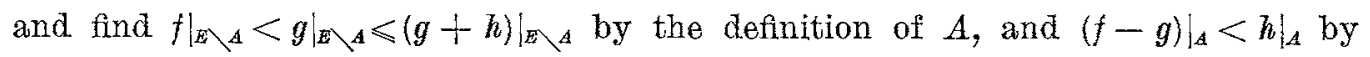
the construction. Hence $f<g+h$.

\section{4. - Scales.}

As in the preceding section, $E$ is a non-empty compact Hausdorff space and $W$ is a subspace of Cont $(E)$ satisfying $\left(W_{1}\right)$.

In this section we introduce a useful technical concept. A $W$-scale is a subset $S$ of $W^{+}$satisfying the following conditions:

$\left(\mathbf{S}_{1}\right) \quad S$ is totally strictly-ordered by $<$; i.e., for all $u, v \in S$, either $u=v$ or $u<v$ or $v<u$;

$\left(\mathbb{S}_{2}\right)$ for each $p \in E$ and $\alpha \in \boldsymbol{P} \times$ there is $u \in S$ such that $u(p)=\alpha$. 
The $u$ occurring in $\left(\mathrm{S}_{2}\right)$ is of course unique. As an important example, the set $\boldsymbol{P}^{\times} w$ is a $W$-scale for each $w \in W^{+}$.

Let $S$ be a $W$-scale. For each $p \in E$ we let $e v_{p}^{s}: S \rightarrow \boldsymbol{P} \times$ be the evaluation mapping defined by $\mathrm{ev}_{p}^{s}(u):=u(p)$ for all $u \in S$. By $\left(\mathrm{S}_{1}\right)$ and $\left(\mathrm{S}_{2}\right), \mathrm{ev}_{p}^{S}$ is a $<-<$-isomorphism (for the restriction of $<$ to $S$ and the usual strict-order $<$ on $\boldsymbol{P}^{\times}$) and hence $a \leqslant-\leqslant-$ isomorphism. It follows that every non-empty subset $T$ of $S$ that has an upper [a lower] $\leqslant$-bound is $S$ also has a least upper [a greatest lower] $\leqslant$-bound in $S$, to be denoted by $\sup _{S} T\left[\inf _{S} T\right]$. It is convenient to use the "Dedekind-Cut Principle" that follows from this fact in the usual manner.

4.1. Proposition. - Let $S$ be a $W$-scale. If the subset $T$ of $S$ satisfies $T \neq \emptyset \neq$ $\neq S \backslash T$ and for every $f \in T$ and $g \in S \backslash T$ we have $f<g$, then there exists a unique $h \in S$ such that $f \leqslant h \leqslant g$ for all $f \in T$ and $g \in S \backslash T$.

4.2. Proposition. - Let $S$ be a $W$-scale, and let $f \in W^{+}$be given. Then:

(a) there exist $u, v \in S$ such that $u<f<v$;

(b) for given $u \in S$ such that $u<f$ there exists $w \in S$ such that $u<w<f$;

(c) for given $v \in S$ such that $f<v$ there exists $w \in S$ such that $f<w<v$.

Proof. - We prove (b), the proof of (a) and (c) being similar. Since $S$ is a $W$-scale, the collection $\{\{p \in E \mid g(p)<f(p)\} \mid g \in S, u<g\}$ is an open covering of $E$; by $\left(\mathbb{S}_{\mathbf{1}}\right)$ this collection is totally ordered by inclusion. There exists, therefore, an element $w \in S$ such that $u<w$ and $\{p \in E \mid w(p)<f(p)\}=E$, i.e., $w<f$.

For a given $W$-scale $S$ we consider the mapping $\omega_{S}: E \times E \times \boldsymbol{P}^{\times} \rightarrow \boldsymbol{P}^{\times}$defined by

$$
\omega_{S}(p, q, \cdot):=\mathrm{ev}_{q}^{S} \circ\left(\mathrm{ev}_{p}^{S}\right)^{-1} \quad \text { for all } p, q \in E \text {; }
$$

this mapping is characterized by the equation

$$
u(q)=\omega_{S}(p, q, u(p)) \quad \text { for all } u \in S \text { and } p, q \in E
$$

4.3. Propostition. - For each $W$-scale $S$ the mapping $\omega_{S}$ is continuous.

Let $\left(p_{0}, q_{0}, \alpha_{0}\right) \in E \times E \times \boldsymbol{P} \times$ and $\left.\varepsilon \in\right] 0,1\left[\right.$ be given. Set $u_{0}:=\left(\mathrm{ev}_{p_{0}}^{S}\right)^{-1}\left(\alpha_{0}\right)$; then

$$
u_{0}\left(p_{0}\right)=\alpha_{0}, \quad u_{0}\left(q_{0}\right)=\omega_{S}\left(p_{0}, q_{0}, \alpha_{0}\right)
$$

Now $0<(1-\varepsilon) u_{0}<u_{0}<(1+\varepsilon) u_{0}$; by Proposition 4.2, (b), (c), we may choose $v$, $w \in S$ such that $(1-\varepsilon) u_{0}<v<u_{0}<w<(1+\varepsilon) u_{0}$. Together with (4.3), this implies $v\left(p_{0}\right)<\alpha_{0}<w\left(p_{0}\right)$ and

$$
(1-\varepsilon) \omega_{S}\left(p_{0}, q_{0}, \alpha_{0}\right)<v\left(q_{0}\right)<w\left(q_{0}\right)<(1+\varepsilon) \omega_{S}\left(p_{0}, q_{0}, \alpha_{0}\right) .
$$


We may choose $\eta \in] 0, \alpha_{0}[$ such that

$$
v\left(p_{0}\right)<\alpha_{0}-\eta<\alpha_{0}+\eta<w\left(p_{0}\right) .
$$

From (4.5) and (4.4) it follows that we may choose neighbourhoods $U$ of $p_{0}$ and $V$ of $q_{0}$ in $E$ such that

$$
\begin{aligned}
v(p)<\alpha_{0}-\eta, & \alpha_{0}+\eta<w(p) \quad \text { for all } p \in U \\
(1-\varepsilon) \omega_{s}\left(p_{0}, q_{0}, \alpha_{0}\right)<v(q), & w(q)<(1+\varepsilon) \omega_{S}\left(p_{0}, q_{0}, \alpha_{0}\right) \quad \text { for all } q \in V .
\end{aligned}
$$

Now $U \times V \times] \alpha_{0}-\eta, \alpha_{0}+\eta\left[\right.$ is a neighbourhood of $\left(p_{0}, q_{0}, \alpha_{0}\right)$ in $E \times E \times \boldsymbol{P} \times$. Suppose that $(p, q, \alpha)$ is in this neighbourhood, and set $u:=\left(e v_{p}^{s}\right)^{-1}(\alpha)$. We have, using (4.2) and (4.6),

$$
\begin{aligned}
& u(p)=\alpha, \quad u(q)=\omega_{s}(p, q, \alpha), \\
& v(p)<\alpha_{0}-\eta<u(p)<\alpha_{0}+\eta<w(p) .
\end{aligned}
$$

Since $u, v, w \in S$, we must have $v<u<w$, and hence, by (4.7),

$$
(1-\varepsilon) \omega_{S}\left(p_{0}, q_{0}, \alpha_{0}\right)<v(q)<\omega_{S}(p, q, \alpha)<w(q)<(1+\varepsilon) \omega_{S}\left(p_{0}, q_{0}, \alpha_{0}\right)
$$

Since $\left(p_{0}, q_{0}, \alpha_{0}\right) \in E \times E \times \boldsymbol{P}^{\times}$and $\left.\varepsilon \in\right] 0,1\left[\right.$ were arbitrary, we conclude that $\omega_{S}$ is continuous.

We now use a $W$-scale to examine more closely the values of the functions in $W^{+}$ on the sets in $\xi(W)$. Let $S$ be a $W$-scale. For each $f \in W^{+}$and each $Z \in Z(W)$, Proposition 4.2 , (a) allows us to define

$$
\begin{aligned}
m_{S}^{+}(f, Z) & :=\inf _{S}\left\{u \in S|f|_{Z} \leqslant\left. u\right|_{Z}\right\} \\
m_{S}^{-}(f, Z) & :=\sup _{S}\left\{u \in S|u|_{Z} \leqslant\left. f\right|_{z}\right\}
\end{aligned}
$$

4.4. Proposttion. - Let \& be a $W$-scale, and let $f \in W^{+}$and $Z \in 3(W)$ be given. Then $\left.f\right|_{Z} \leqslant\left. m_{S}^{+}(f, Z)\right|_{Z}$, with $f(p)=\left(m_{S}^{+}(f, Z)\right)(p)$ for some $p \in Z$; and $\left.m_{S}^{-}(f, Z)\right|_{Z} \leqslant\left. f\right|_{Z}$, with $\left(m_{S}^{-}(f, Z)\right)(p)=f(p)$ for some $p \in Z$.

Proof. - Suppose that $p \in Z$ were such that $\left(m_{S}^{+}(f, Z)\right)(p)<f(p)$. By $\left(\mathbb{S}_{2}\right)$ we may choose $u \in S$ such that $\left(m_{S}^{+}(f, Z)\right)(p)<u(p)<f(p)$, and by $\left(\mathbb{S}_{1}\right)$ we have $m_{S}^{+}(f, Z)<u$. But $\left(S_{1}\right)$ and the definition of $m_{S}^{+}(f, Z)$ together then require $f(p) \leqslant u(p)$, a contradiction. Therefore no such $p$ exists, and $\left.f\right|_{Z} \leqslant\left. m_{s}^{+}(f, Z)\right|_{z}$ :

Suppose that $\left.f\right|_{Z}<\left.m_{S}^{+}(f, Z)\right|_{Z}$; since $Z$ is non-empty, and compact by Proposition 3.1, we have

$$
\left.\alpha:=\max \frac{\left.f\right|_{z}}{\left.m_{s}^{+}(f, Z)\right|_{z}} \in\right] 0,1[
$$


Then $\alpha m_{S}^{+}(f, Z)<m_{S}^{+}(f, Z)$, and by Proposition 4.2, (c) we may choose $w \in S$ such that $\alpha m_{S}^{+}(f, Z)<w<m_{S}^{+}(f, Z)$. But then $\left.f\right|_{Z} \leqslant\left.\alpha m_{S}^{+}(f, Z)\right|_{Z}<\left.w\right|_{Z}$, whence $m_{S}^{+}(f, Z)<w$, a contradiction. Therefore $f(p)=\left(m_{S}^{+}(f, Z)\right)(p)$ for some $p \in Z$.

The proof of the assertion about $m_{s}^{-}(f, Z)$ is entirely analogous.

4.5. Corollary. - Let $S$ be a $W$-scale, and let $f \in W^{+}, u \in S$, and $Z \in \mathcal{Z}(W)$ be given. Then $m_{S}^{+}(f, Z)<u$ if and only if $\left.f\right|_{Z}<\left.u\right|_{Z}$ and $u<m_{S}^{-}(f, Z)$ if and only if $\left.u\right|_{Z}<\left.f\right|_{Z}$ :

4.6. Proposition. - Let $S$ be a $W$-scale, and let $f \in W^{+}, u \in S$, and $Z \in Z(W)$ be given. Then $u<m_{S}^{-}(f, Z)$ if and only if there exists $g \in W^{+}$such that $u<g$ and $f \leqslant g$ and $m_{S}^{+}(g, Z) \leqslant m_{S}^{+}(f, Z)$.

Proof. - Assume that $u<m_{S}^{-}(f, Z)$. By Corollary 4.5, $\left.u\right|_{z}<\left.f\right|_{z}$. By Proposition 3.6, we may choose $h \in J_{W}(Z)$ such that $u<f+h$. By Proposition 4.4, $\left.(f+h)\right|_{z}=$ $=\left.f\right|_{z} \leqslant\left. m_{S}^{+}(f, Z)\right|_{z}$, and hence $m_{S}^{+}(f+h, Z) \leqslant m_{S}^{+}(f, Z)$. Thus $g:=f+h$ satisfies all the requirements of the statement.

Assume, conversely, that $g \in W^{+}$satisfies $u<g$ and $f \leqslant g$ and $m_{S}^{+}(g, Z) \leqslant m_{S}^{+}(f, Z)$. By Proposition $4.4,\left.f\right|_{z} \leqslant\left. g\right|_{z} \leqslant\left. m_{S}^{+}(g, Z)\right|_{z} \leqslant\left. m_{S}^{+}(f, Z)\right|_{z}$, and we may choose $p \in Z$ such that $f(p)=\left(m_{S}^{+}(f, Z)\right)(p)$; hence $f(p)=g(p)$. Now $g-f \in \bar{W}+$ and $(g-f)(p)=0$. By $\left(Z_{1}\right),\left.(g-f)\right|_{z}=0$, and hence $\left.u\right|_{z}<\left.g\right|_{z}=\left.f\right|_{z}$. By Corollary 4.5, $u<m_{S}^{-\bar{s}}(f, Z)$.

Our final result in this section gives some additional information when $W$ satisfies the "separation condition» $\left(\mathrm{W}_{\mathrm{a}}\right)$.

4.7. Proposition. - Assume that $W$ satisfies $\left(W_{2}\right)$. Let $S$ be a $W$-scale, and let $Z \in 3(W)$ be given. Then $m_{s}^{-}(f, Z)=m_{S}^{+}(f . Z)$ for all $f \in W^{+}$if and only if $Z$ is a singleton.

Proof. - If $Z$ is a singleton, say $Z=\{p\}$, and $f \in W^{+}$, Proposition 4.4 implies $\left(m_{S}^{-}(f, Z)\right)(p)=f(p)=\left(m_{S}^{+}(f, Z)\right)(p)$. Since $S$ is a $W$-scale, this implies $m_{S}^{-}(f, Z)=$ $=m_{S}^{+}(f, Z)$.

If $Z$ is not a singleton, choose distinct points $p, q \in Z$. Choose $u \in S$ and, in view of $\left(\mathrm{W}_{2}\right)$, choose $w \in W$ such that $w(p)=0<w(q)$. Set

$$
f:=u+\frac{1}{1+\max } \overline{(|w| / u)} w .
$$

We find $f \in W^{+}, f(p)=u(p), f(q)>u(q)$. Then $\left(m_{s}^{-}(f, Z)\right)(p) \leqslant u(p),\left(m_{S}^{+}(f, Z)\right)(q)>$ $>u(q)$, whence $m_{s}^{-}(f, Z) \leqslant u<m_{s}^{+}(f, Z)$.

\section{5. - Structures of order-isomorphisms: preliminaries.}

In this section we assume that $E$ and $E^{\prime}$ are non-empty compact Hausdorff spaces, and that $W$ and $W^{\prime}$ are subspaces of Cont $(E)$ and Cont $\left(E^{\prime}\right)$, respectively, and that both satisfy $\left(\mathrm{W}_{1}\right)$. 
We are interested in studying the $\leqslant-\leqslant$-isomorphisms and $<-<$-isomorphisms from $W^{+}$to $W^{\prime+}$. We observe that, on account of $(2.2)$, every $<-<$-isomorphism is also $a \leqslant-\leqslant$-isomorphism. The converse implication, however, is false in general: see the Remark after Theorem 6.5. A cone-isomorphism from $W^{+}$to $W^{\prime}$ is, by (2.1), also a $<-<$-isomorphism, and hence a $\leqslant-\leqslant$-isomorphism: cone-isomorphisms are, in a sense, the ideal order-isomorphisms (see Section 8).

Let $\varphi: W^{+} \rightarrow W^{\prime+}$ be a $\leqslant-\leqslant$-isomorphism. Since we do not know whether $\varphi$ is a $<-<$-isomorphism, we define the set

$$
W_{\varphi}^{+}:=\left\{f \in W^{+} \mid g<f \Leftrightarrow \varphi(g)<\varphi(f) \text { and } f<g \Leftrightarrow \varphi(f)<\varphi(g) \text { for all } g \in W^{+}\right\} \text {. }
$$

We note that $\varphi$ is a $<-<$-isomorphism if and only if $W_{\varphi}^{+}=W^{+}$. We are unable to analyse the structure of $\varphi$ unless $W_{\varphi}^{+}$is fairly extensive; to obtain a criterion for this, we establish a result about scales. We say that the $W$-scale $S$ scales $\varphi$ if $\varphi_{>}(S)$ is a $W^{\prime}$-scale; we say that $\varphi$ is scaled if some $W$-scale scales $\varphi$.

5.1. Proposimion. - Let $\varphi: W^{+} \rightarrow W^{\prime+}$ be $a \leqslant-\leqslant-$ isomorphism and $S$ a $W$-scale Then $S$ scales $\varphi$ if and only if $S \subset W_{\varphi}^{+}$.

Proof. - The condition is necessary. Suppose that $S^{\prime}:=\varphi_{>}(S)$ is a $W^{\prime}$-scale. Let $u \in S$ and $f \in W^{+}$be given. Suppose that $f<u$. By Proposition 4.2,(c) we may choose $w \in S$ such that $f<w<u$. Now $f \leqslant w \leqslant u$ and $w \neq u$, whence $\varphi(f) \leqslant \varphi(w) \leqslant$ $\leqslant \varphi(u)$ and $\varphi(w) \neq \varphi(u)$. Since $S^{r}$ is a $W^{\prime}$-scale, we must have $\varphi(w)<\varphi(u)$, and hence $\varphi(f)<\varphi(u)$. Since $S=\left(\varphi^{-1}\right)_{>}\left(S^{\prime}\right)$, the same argument, with $\varphi, S, u, f$ replaced by $\varphi^{-1}, S^{\prime}, \varphi(u), \varphi(f)$, respectively, shows that $\varphi(f)<\varphi(u)$ implies $f<u$. Thus $f<u \Leftrightarrow$ $\Leftrightarrow \varphi(f)<\varphi(u)$.

A similar argument, using Proposition 4.2,(b), establishes the equivalence $u<t \Leftrightarrow$ $\Leftrightarrow \varphi(u)<\varphi(f)$. Since $f \in W^{+}$was arbitrary, it follows that $u \in W_{\varphi}^{+}$. Since $u \in S$ was arbitrary, we find that $S \subset W_{\varphi}^{+}$.

The condition is sufficient. Suppose that $S \subset W_{\varphi}^{+}$. Let $u, v \in S$ be given, with $u<v$. By the assumption, $\varphi(u)<\varphi(v)$. Since $S$ satisfies $\left(\mathbf{S}_{1}\right)$, it follows that $S^{\prime \prime}:=\varphi_{>}(S)$ does so too.

Let $p^{\prime} \in E^{\prime}$ and $\alpha^{\prime} \in \boldsymbol{P} \times$ be given, and suppose that $(\varphi(f))\left(p^{\prime}\right) \neq \alpha^{\prime}$ for all $f \in S$. Set $T:=\left\{f \in S \mid(\varphi(f))\left(p^{\prime}\right)<\alpha^{\prime}\right\}$; then $S \backslash T=\left\{f \in S \mid(\varphi(f))\left(p^{\prime}\right)>\alpha^{\prime}\right\}$. For every $f \in T$ and $g \in \mathbb{S} \backslash T$ we have $(\varphi(f))\left(p^{\prime}\right)<(\varphi(g))\left(p^{\prime}\right)$, whence $\varphi(f)<\varphi(g)$ since $S^{\prime}$ satisfies $\left(\mathbf{S}_{\mathbf{1}}\right)$; by the assumption, this implies $f<g$.

Choose $w^{\prime} \in W^{\prime}+$; replacing $w^{\prime}$ by $\left(\alpha^{\prime} / w^{\prime}\left(p^{\prime}\right)\right) w^{\prime}$, if necessary, we may and do assume that $w^{\prime}\left(p^{\prime}\right)=\alpha^{\prime}$. By Proposition 4.2,(a), we may choose $u, v \in S$ such that $u<\varphi^{-1}\left(w^{\prime}\right)<v$; by the assumption, this implies $\varphi(u)<w^{\prime}<\varphi(v)$ and hence $(\varphi(u))\left(p^{\prime}\right)<\alpha^{\prime}<(\varphi(v))\left(p^{\prime}\right)$; hence $u \in T$ and $v \in S \backslash T$. By Proposition 4.1, there exists a unique $h \in \mathcal{S}$ such that $f \leqslant h \leqslant g$ for all $f \in T$ and $g \in \mathcal{S} \backslash T$. Set $\beta^{\prime}:=(\varphi(h))\left(p^{\prime}\right) \in \boldsymbol{P} \times$.

Suppose that $h \in T$, so that $\beta^{\prime}<\alpha^{\prime}$. Then $\varphi(h)<\left(\alpha^{\prime} \mid \beta^{\prime}\right) \varphi(h)$; by the assumption, $h<\varphi^{-1}\left(\left(\alpha^{\prime} \mid \beta^{\prime}\right) \varphi(h)\right)$. By Proposition 4.2(b), we may choose $w \in S$ such that 
$h<w<\varphi^{-1}\left(\left(\alpha^{\prime} \mid \beta^{\prime}\right) \varphi(h)\right)$. Then $\varphi(w)<\left(\alpha^{\prime} \mid \beta^{\prime}\right) \varphi(h)$, and hence $(\varphi(w))\left(p^{\prime}\right)<\alpha^{\prime} ;$ this implies $w \in T$, so that $w \leqslant h$, a contradiction.

Suppose, on the other hand, that $h \in S \backslash T$, so that $\alpha^{\prime}<\beta^{\prime}$. In a similar manner, and using Proposition 4.2(c), we may choose $w \in S$ such that $\varphi^{-1}\left(\left(\alpha^{\prime} / \beta^{\prime}\right) \varphi(h)\right)<$ $<w<h$. Then $\left(\alpha^{\prime} \mid \beta^{\prime}\right) \varphi(h)<\varphi(w)$ and hence $\alpha^{\prime}<(\varphi(w))\left(p^{\prime}\right)$; this implies $w \in S \backslash T$, so that $h \leqslant w$, a contradiction.

The hypothesis that $(\varphi(f))\left(p^{\prime}\right) \neq \alpha^{\prime}$ for all $f \in S$ is thus untenable. Since $p^{\prime} \in E^{\prime}$ and $\alpha^{\prime} \in \boldsymbol{P}^{\times}$were arbitrary, we conclude that $\boldsymbol{S}^{\prime}$ also satisfies $\left(\mathbf{S}_{2}\right)$ and is thus a $W^{\prime}$ scale.

5.2. Corollary. $-A<-<$-isomorphism $\varphi: W^{+} \rightarrow W^{\prime+}$ is necessarily a scaled $\leqslant-\leqslant-$ isomorphism; it is sealed by every $W$-scale.

Proof. - We already noted that $\varphi$ is a $\leqslant-\leqslant-$-isomorphism (by (2.2)), and that $W_{p}^{+}=W^{+}$. Since $W$ satisfies $\left(W_{1}\right)$ we may choose $w \in W^{+}$. Then $\boldsymbol{P} \times w$ is a $W$-scale. It follows from Proposition 5.1 that every $W$-scale, and in particular $\boldsymbol{P} \times w$, scales $p$.

Our main objective in this section is to prove the following proposition and an important application of it.

5.3. Proposttion. - Let $\varphi: W^{+} \rightarrow W^{\prime+}$ be a scaled $\leqslant-\leqslant-i s o m o r p h i s m$ (in particular, a<-<-isomorphism). Then there exists a unique bijection $\Sigma: 3\left(W^{\prime}\right) \rightarrow 3(W)$ such that

$$
\varphi(u)+J_{W^{\prime}}\left(Z^{\prime}\right)=\varphi_{>}\left(u+J_{W}\left(\Sigma\left(Z^{\prime}\right)\right)\right) \quad \text { for all } u \in W_{\varphi} \text { and } Z^{\prime} \in \mathcal{Z}\left(W^{\prime}\right) .
$$

We first have to establish a weaker form of this assertion.

5.4. LEMMa. - Let $\varphi: W^{+} \rightarrow W^{\prime+}$ be a $\leqslant-\leqslant-$ isomorphism. For every $u \in W_{\varphi}^{+}$ there is a unique bijection $\Sigma_{u}: \zeta\left(W^{\prime}\right) \rightarrow 3(W)$ such that

$$
\varphi(u)+J_{W^{\prime}}\left(Z^{\prime}\right)=\varphi_{>}\left(u+J_{W}\left(\Sigma_{u}\left(Z^{\prime}\right)\right)\right) \quad \text { for all } Z^{\prime} \in \mathcal{Z}\left(W^{\prime}\right)
$$

Proof. - Let $u \in W_{\varphi}^{+}$be given. Since $\varphi$ is a $\leqslant-\leqslant$-isomorphism, we have, by (2.1), $\varphi_{>}\left(u+\bar{W}^{+}\right)=\varphi(u)+\bar{W}^{\prime+} ;$ by the definition of $W_{\varphi}^{+}$and $(2.1)$ we also have $\varphi_{>}(u+$ $\left.+W^{+}\right)=\varphi(u)+W^{\prime+}$; and hence $\varphi_{>}\left(u+\bar{W}^{+} \backslash W^{+}\right)=\varphi(u)+\bar{W}^{\prime+} \backslash W^{\prime+}$.

The mapping $\psi_{u}: \bar{W}^{+} \backslash W^{+} \rightarrow \bar{W}^{\prime} \backslash \backslash W^{\prime+}$ defined by

$$
\psi_{u}(f):=\varphi(u+f)-\varphi(u) \quad \text { for all } f \in \bar{W}^{+} \backslash W^{+}
$$

is therefore well defined and $a \leqslant-\leqslant$-isomorphism (it satisfies $\left(\psi_{u}\right)^{-1}\left(f^{\prime}\right)=\varphi^{-1}(\varphi(u)+$ $\left.\left.+f^{\prime}\right)-u\right)$. Consequently, the mapping $\left(\psi_{u}\right)_{>}: \mathfrak{P}\left(\bar{W}^{+} \backslash W^{+}\right) \rightarrow \mathfrak{H}\left(\bar{W}^{\prime} \backslash \backslash W^{\prime}\right)$ has a restriction to a bijection from the collection of all maximal $\leqslant$-directed subsets of $\bar{W}^{+} \backslash W^{+}$to the collection of all maximal $\leqslant$-directed subsets of $\bar{W}^{\prime} \backslash \bar{W}^{\prime}$. 
By Proposition 3.2 this implies the existence of a unique bijection $\Sigma_{u}: 3\left(W^{\prime}\right) \rightarrow$ $\rightarrow \mathfrak{Z}(W)$ such that

$$
J_{W}\left(Z^{\prime}\right)=\left(\psi_{u}\right)_{>}\left(J_{W}\left(\Sigma_{u}\left(Z^{\prime}\right)\right)\right) \quad \text { for all } Z^{\prime} \in \mathcal{Z}\left(W^{\prime}\right)
$$

The assertion follows from this and (5.2).

Proof of Proposition 5.3. - It is clearly enough to show that $\Sigma_{u}=\Sigma_{v}$ for all $u$, $v \in W_{\varphi}$, where $\Sigma_{u}, \Sigma_{v}$ are as in Lemma 5.4. If the $W$-scale $S$ scales $\varphi$, then $S \subset W_{\varphi}^{+}$ by Proposition 5.1, and for given $u, v \in W_{\varphi}^{+}$we may choose $w \in S \subset W_{\varphi}^{+}$such that $u+v<w$ by Proposition 4.2,(a), whence $u<w$ and $v<w$. We infer from this remark that it will be enough to show that $\Sigma_{u}=\Sigma_{v}$ for all $u, v \in W_{\varphi}^{+}$with $u<v$.

Let then $u, v \in W_{\varphi}^{+}$be given, with $u<v$, and let $Z^{\prime} \in \tilde{3}\left(W^{\prime}\right)$ be given. Suppose that $\Sigma_{u}\left(Z^{\prime}\right) \neq \Sigma_{v}\left(Z^{\prime}\right)$. By Proposition 3.1, $\Sigma_{u}\left(Z^{\prime}\right)$ and $\Sigma_{v}\left(Z^{\prime}\right)$ are disjoint non-empty sets. Choose $q \in \Sigma_{v}\left(Z^{\prime}\right)$. By $\left(Z_{2}\right)$ we may choose $f \in J_{W}\left(\Sigma_{u}\left(Z^{\prime}\right)\right)$ such that $f(q)>0$; replacing $f$ by a suitable positive scalar multiple, if necessary, we may assume without loss that

$$
f(q)>v(q)-u(q)
$$

Now $u+f \in u+J_{W}\left(\Sigma_{u}\left(Z^{\prime}\right)\right)$ and hence, by (5.1), $\varphi(u+f) \in \varphi(u)+J_{W^{\prime}}\left(Z^{\prime}\right)$. But then $\varphi(v)+\varphi(u+f)-\varphi(u) \in \varphi(v)+J_{W^{\prime}}\left(Z^{\prime}\right)$; by (5.1) again, $\varphi^{-1}(\varphi(v)+\varphi(u+f)-$ $-\varphi(u)) \in v+J_{W}\left(\Sigma_{v}\left(Z^{\prime}\right)\right)$. Set $g:=\varphi^{-1}(\varphi(v)+\varphi(u+f)-\varphi(u))$. We have shown that $g(q)=v(q)$; moreover, $\varphi(u)<\varphi(v)$, whence $\varphi(u+f)<\varphi(g)$, whence $u+f \leqslant g$. This however, implies $u(q)+f(q) \leqslant g(q)=v(q)$, which contradicts (5.3). Our supposition that $\Sigma_{u}\left(Z^{\prime}\right) \neq \Sigma_{v}\left(Z^{\prime}\right)$ is therefore untenable, and the proof is complete.

5.5. Proposition. - Let $\varphi: W^{+} \rightarrow W^{+}$be a $\leqslant-\leqslant-$isomorphism scaled by the $W$ scale $S$. For every $f \in W^{+}$and $Z^{\prime} \in \mathcal{Z}\left(W^{\prime}\right)$ we have

$$
\begin{aligned}
& m_{S^{\prime}}^{+}\left(\varphi(f), Z^{\prime}\right)=\varphi\left(m_{S}^{+}\left(f, \Sigma\left(Z^{\prime}\right)\right)\right), \\
& m_{S^{\prime}}^{-}\left(\varphi(f), Z^{\prime}\right)=\varphi\left(m_{s}^{-}\left(f, \Sigma\left(Z^{\prime}\right)\right)\right),
\end{aligned}
$$

where $S^{\prime}:=\varphi_{>}(S)$.

1. Let $f \in W^{+}$and $Z^{\prime} \in \mathcal{Z}\left(W^{\prime}\right)$ be given. Let $u \in S^{\prime}$ satisfy $m_{S}^{+}\left(f, \Sigma\left(Z^{\prime}\right)\right)<u$. By Corollary 4.5, $\left.f\right|_{\Sigma\left(Z^{\prime}\right)}<\left.u\right|_{\Sigma\left(Z^{\prime}\right)}$. By Proposition 3.6 we may choose $g \in u+J_{W}\left(\Sigma\left(Z^{\prime}\right)\right)$ such that $f<g$. Then $\varphi(f) \leqslant \varphi(g)$ and, by Proposition 5.3, $\varphi(g) \in \varphi(u)+J_{W^{\prime}}\left(Z^{\prime}\right)$. Hence $\left.\varphi(f)\right|_{Z^{\prime}} \leqslant\left.\varphi(g)\right|_{Z^{\prime}}=\left.\varphi(u)\right|_{Z^{\prime}}$; since $\varphi(u) \in S^{\prime}$, it follows that $m_{S^{\prime}}^{+}\left(\varphi(f), Z^{\prime}\right) \leqslant \varphi(u)$. We have shown that, for every $u \in S, m\left({ }_{s}^{+},, \Sigma\left(Z^{\prime}\right)\right)<u$ implies $m_{S^{\prime}}^{+}\left(\varphi(f), Z^{\prime}\right) \leqslant \varphi(u)$. By the same argument applied to $\varphi^{-1}$ it follows that $m_{S^{\prime}}^{+}\left(\varphi(f), Z^{\prime}\right)<\varphi(u)$ implies $m_{S}^{+}\left(f, \Sigma\left(Z^{\prime}\right)\right) \leqslant u$. Since $\varphi$ has a restriction to a $<-<$-isomorphism from $S$ to $S^{\prime}$, the equation (5.4) follows. 
2. The proof of (5.5) is not analogous to that of (5.4), and in fact uses the latter equation. Let $f \in W^{+}$and $Z^{\prime} \in \mathcal{Z}\left(W^{\prime}\right)$ again be given. Let $u \in S$ satisfy $u<m_{S}^{-}(f$, $\left.\Sigma\left(Z^{\prime}\right)\right)$. By Proposition 4.6 we may choose $g \in W^{+}$such that $u<g$ and $f \leqslant g$ and $m_{s}^{+}\left(g, \Sigma\left(Z^{\prime}\right)\right) \leqslant m_{s}^{+}\left(f, \Sigma\left(Z^{\prime}\right)\right)$. By the assumptions on $\varphi$ and $S$, by Proposition 5.1, and by (5.4), we have $\varphi(u)<\varphi(g)$ and $\varphi(f) \leqslant \varphi(g)$ and $m_{S^{\prime}}^{+}\left(\varphi(g), Z^{\prime}\right) \leqslant m_{S^{\prime}}^{+}\left(\varphi(f), Z^{\prime}\right)$. By Proposition 4.6 we conclude that $\varphi(u)<m_{\mathbb{S}^{\prime}}^{-}\left(\varphi(f), Z^{\prime}\right)$. We have shown that for every $u \in S, u<m_{S}^{-}\left(f, \Sigma\left(Z^{\prime}\right)\right)$ implies $\varphi(u)<m_{s^{\prime}}^{-}\left(\varphi(f), Z^{\prime}\right)$. The reverse implication follows by the same argument applied to $\varphi^{-1}$. Since $\varphi$ has a restriction to a $<-<$-isomorphism from $S$ to $S^{\prime}$, equation (5.5) follows.

\section{6. - Structure of order-isomorphisms: main results.}

As in the preceding section, we assume that $E$ and $E^{\prime}$ are nonempty compact Hausdorff spaces, and that $W$ and $W^{\prime}$ are subspaces of Cont $(E)$ and Cont $\left(E^{\prime}\right)$, respectively, and that both satisfy $\left(\mathrm{W}_{1}\right)$. We assume, in addition, that $W$ and $W^{\prime}$ both satisfy $\left(\mathrm{W}_{2}\right)$, the "separation condition". (Cf. Proposition 2.1.).

Our purpose in this section is to examine the effect of a scaled $\leqslant-\leqslant$-isomorphism from $W^{+}$to $W^{\prime}$ on the values at points of $P(W)$ and $P\left(W^{\prime}\right)$ (defined in Section 2) of elements of $W^{+}$and their images. In the sequel, $P(W)$ and $P\left(W^{\prime}\right)$ are considered to be equipped with the subspace topologies induced by $E$ and $E^{\prime}$, respectively.

6.1. Theorem. - Assume that $\varphi: W^{+} \rightarrow W^{\prime+}$ is a scaled $\leqslant-\leqslant-$ isomorphism (in particular, a $<-<-$-isomorphism). Then:

(a) There exists a unique combination of a bijection $\sigma: P\left(W^{\prime}\right) \rightarrow P(W)$ and a family $\left(\gamma_{p^{\prime}} \mid p^{\prime} \in P\left(W^{\prime}\right)\right)$ of $<-<-$ isomorphisms from $\boldsymbol{P}^{\times}$to $\boldsymbol{P}^{\times}$such that

$$
(\varphi(f))\left(p^{\prime}\right)=\gamma_{p^{\prime}}\left(f\left(\sigma\left(p^{\prime}\right)\right)\right) \quad \text { for all } f \in W^{+} \text {and } p^{\prime} \in P\left(W^{\prime}\right) .
$$

(b) $\sigma$ and the mapping $\Gamma: P\left(W^{\prime}\right) \times \boldsymbol{P}^{\times} \rightarrow P\left(W^{\prime}\right) \times \boldsymbol{P}^{\times}$defined by

$$
\Gamma\left(p^{\prime}, \alpha\right):=\left(p^{\prime}, \gamma_{p^{\prime}}(\alpha)\right) \quad \text { for all }\left(p^{\prime}, \alpha\right) \in P\left(W^{\prime}\right) \times \boldsymbol{P}^{\times}
$$

are homeomorphisms.

Proof. - Proof of (a). We first prove the uniqueness assertion. Suppose that $\sigma^{1}$, $\sigma^{2}: P\left(W^{\prime}\right) \rightarrow P(W)$ are bijections and, for every $p^{\prime} \in P\left(W^{\prime}\right), \gamma_{p^{\prime}}^{1}, \gamma_{p^{\prime}}^{2}: \boldsymbol{P}^{\times} \rightarrow \boldsymbol{P}^{\times}$are $<-<$-isomorphisms, and that (6.1) holds for $\sigma:=\sigma^{i}, \gamma_{p^{\prime}}:=\gamma_{p^{\prime}}^{i}, i=1,2$. Suppose $p^{\prime} \in P\left(W^{\prime}\right)$ is such that $\sigma^{1}\left(p^{\prime}\right) \neq \sigma^{2}\left(p^{\prime}\right)$. We choose $w \in W^{+}$. Since $\sigma^{1}\left(p^{\prime}\right) \in P(W)$ we may, by (3.1), choose $g \in \overline{W^{+}}$such that $g\left(\sigma^{1}\left(p^{\prime}\right)\right)=0<g\left(\sigma^{2}\left(p^{\prime}\right)\right)$. Then $w+g \in W^{+}$ and $w\left(\sigma^{2}\left(p^{\prime}\right)\right)<(w+g)\left(\sigma^{2}\left(p^{\prime}\right)\right)$; hence, by $(6.1)$,

$$
\begin{aligned}
(\varphi(w))\left(p^{\prime}\right) & =\gamma_{p^{\prime}}^{2}\left(w\left(\sigma^{2}\left(p^{\prime}\right)\right)\right)<\gamma_{p^{\prime}}^{2}\left((w+g)\left(\sigma^{2}\left(p^{\prime}\right)\right)\right)=(\varphi(w+g))\left(p^{\prime}\right)= \\
& =\gamma_{p^{\prime}}^{1}\left((w+g)\left(\sigma^{1}\left(p^{\prime}\right)\right)\right)=\gamma_{p^{\prime}}^{\mathbf{1}}\left(w\left(\sigma^{1}\left(p^{\prime}\right)\right)\right)=(\varphi(w))\left(p^{\prime}\right),
\end{aligned}
$$


a contradiction. Therefore no such $p^{\prime}$ exists, and $\sigma^{1}=\sigma^{2}$. Since

$$
\left\{f\left(\sigma^{x}\left(p^{\prime}\right)\right) \mid f \in W^{\dagger}\right\}=\boldsymbol{P}^{\times} \quad \text { for each } p^{\prime} \in P\left(W^{\prime}\right),
$$

it follows from (6.1) that $\gamma_{p^{\prime}}^{1}=\gamma_{p^{\prime}}^{2}$ for all $p^{\prime} \in P\left(W^{\prime}\right)$.

We next turn to the existence proof, and begin by examining what action the mapping $\Sigma: \mathfrak{Z}\left(W^{\prime}\right) \rightarrow \mathfrak{Z}(W)$ obtained in the preceding section has on singletons. We choose a $W$-scale $S$ that scales $\varphi$, and set $S^{\prime}:=\varphi_{>}(S)$. Let $Z^{\prime} \in \mathcal{Z}\left(W^{\prime}\right)$ be given. By Proposition $4.7, Z^{\prime}$ is a singleton if and only if $m_{S^{\prime}}^{-}\left(\varphi(f), Z^{\prime}\right)=m_{S^{\prime}}^{+}\left(\varphi(f), Z^{\prime}\right)$ for all $f \in W^{+}$; by Proposition 5.5, this is the case if and only if $m_{S}^{-}\left(f, \Sigma\left(Z^{\prime}\right)\right)=m_{s}^{+}\left(f, \Sigma\left(Z^{\prime}\right)\right)$ for all $f \in W^{+}$; again by Proposition 4.7 , this is in turn the case if and only if $\Sigma\left(Z^{\prime}\right)$ is a singleton. We therefore conclude that there is a unique bijection $\sigma: P\left(W^{\prime}\right) \rightarrow P(W)$ such that

$$
\left\{\sigma\left(p^{\prime}\right)\right\}=\Sigma\left(\left\{p^{\prime}\right\}\right) \quad \text { for all } p^{\prime} \in P\left(W^{\prime}\right)
$$

We shall see that this is the bijection $\sigma$ required for the assertion.

For each $p^{\prime} \in P\left(W^{\prime}\right)$ we define the mapping

$$
\gamma_{p^{\prime}}:=\mathrm{ev}_{p^{\prime}}^{s^{\prime}} \circ\left(\left.\varphi\right|_{s} ^{s^{\prime}}\right) \circ\left(\mathrm{eV}_{\sigma\left(p^{\prime}\right)}^{s}\right)^{-1}: \boldsymbol{P}^{\times} \rightarrow \boldsymbol{P}^{\times} .
$$

This mapping is a $<-<-$ isomorphism (being the composite of three $<-<-$-isomorphisms), and satisfies

$$
(\varphi(u))\left(p^{\prime}\right)=\gamma_{p^{\prime}}\left(u\left(\sigma\left(p^{\prime}\right)\right)\right) \quad \text { for all } u \in S
$$

Let $f \in W^{+}$be given. For every $u \in S$ we have the following chain of equivalences, by Proposition 5.5, (6.3), Proposition 4.4, and (6.5):

$$
\begin{aligned}
(\varphi(f))\left(p^{\prime}\right) \leqslant(\varphi(u))\left(p^{\prime}\right) & \Leftrightarrow m_{s^{\prime}}^{+}\left(\varphi(f),\left\{p^{\prime}\right\}\right) \leqslant \varphi(u) \Leftrightarrow \\
& \Leftrightarrow \varphi\left(m_{s^{\prime}}^{+}\left(f,\left\{\sigma\left(p^{\prime}\right)\right\}\right)\right) \leqslant \varphi(u) \Leftrightarrow m_{S}^{+}\left(f,\left\{\sigma\left(p^{\prime}\right)\right\}\right) \leqslant u \Leftrightarrow \\
& \Leftrightarrow f\left(\sigma\left(p^{\prime}\right)\right) \leqslant u\left(\sigma\left(p^{\prime}\right)\right) \Leftrightarrow \gamma_{p^{\prime}}\left(f\left(\sigma\left(p^{\prime}\right)\right)\right) \leqslant \gamma_{p^{\prime}}\left(u\left(\sigma\left(p^{\prime}\right)\right)\right) \Leftrightarrow \\
& \Leftrightarrow \gamma_{p^{\prime}}\left(f\left(\sigma\left(p^{\prime}\right)\right)\right) \leqslant(\varphi(u))\left(p^{\prime}\right) .
\end{aligned}
$$

Since the $W^{\prime}$-scale $\mathbb{S}^{\prime}$ satisfies $\left(\mathbb{S}_{2}\right)$, this implies that $(\varphi(f))\left(p^{\prime}\right) \leqslant \alpha \Leftrightarrow \gamma_{p^{\prime}}\left(f\left(\sigma\left(p^{\prime}\right)\right)\right) \leqslant \alpha$ for all $\alpha \in \boldsymbol{P} \times$, and therefore $(\varphi(f))\left(p^{\prime}\right)=\gamma_{p^{\prime}}\left(f\left(\sigma\left(p^{\prime}\right)\right)\right)$. Since $p^{\prime} \in P\left(W^{\prime}\right)$ and $f \in W^{+}$ were arbitrary, (6.1) holds.

We remark that the construction of $\gamma_{y^{\prime}}$ depended on the choice of $S$, but the uniqueness part of the assertion shows that the outcome of the construction is the same for all choices of $S$. 
Proof of (b). We again choose a $W$-scale $S$ that scales $\varphi$, and set $S^{\prime}:=\varphi_{>}(S)$.

Let $p^{\prime} \in P\left(W^{\prime}\right)$ and an open neighbourhood $U$ of $\sigma\left(p^{\prime}\right)$ in $E$ be given. By Lemma 3.7 applied to $Z:=\left\{\sigma\left(p^{\prime}\right)\right\}$ and $A:=E \backslash U$ we may choose $k \in J_{W}\left(\left\{\sigma\left(p^{\prime}\right)\right\}\right)$ such that $\left.k\right|_{E \backslash D}>0$. We further choose $u \in S$. By Proposition 4.2,(b), we may choose $v \in S$ such that $u<v<\left(1+\min \left(\left.(k / u)\right|_{\mathbb{Z} \backslash v}\right)\right) u$. Set $V:=\{q \in E \mid(u+h)(q)<v(q)\}$. Then $V$ is an open neighbourhood of $\sigma\left(p^{\prime}\right)$ in $E$, and $V \subset U$; to prove this inclusion, we observe that if $q \in V$ then $k(q)<v(q)-u(q)<\left(\min \left(\left.(k / u)\right|_{V_{\searrow E}}\right)\right) u(q)$, and therefore $q \notin E \backslash U$.

For every $q^{\prime} \in P\left(W^{\prime}\right)$ we have the following chain of equivalences, by Part (a) of this theorem:

$$
\begin{aligned}
\sigma\left(q^{\prime}\right) \in V & \Leftrightarrow(u+k)\left(\sigma\left(q^{\prime}\right)\right)<v\left(\sigma\left(q^{\prime}\right)\right) \Leftrightarrow \\
& \Leftrightarrow \gamma_{q^{\prime}}\left((u+k)\left(\sigma\left(q^{\prime}\right)\right)\right)<\gamma_{q^{\prime}}\left(v\left(\sigma\left(q^{\prime}\right)\right)\right) \Leftrightarrow \\
& \Leftrightarrow(\varphi(u+k))\left(q^{\prime}\right)<(\varphi(v))\left(q^{\prime}\right) .
\end{aligned}
$$

Thus $\sigma^{<}(U \cap P(W)) \supset \sigma^{<}(V \cap P(W))=\left\{q^{\prime} \in P\left(W^{\prime}\right) \mid(\varphi(u+k))\left(q^{\prime}\right)<(\varphi(v))\left(q^{\prime}\right)\right\}$, an open neighbourhood of $p^{\prime}$ in $P\left(W^{\prime}\right)$. Since $p^{\prime} \in P\left(W^{\prime}\right)$ and the open neighbourhood $U$ of $\sigma\left(p^{\prime}\right)$ in $E$ were arbitrary, we conclude that $\sigma$ is continuous.

Applying the same proof to $\varphi^{-1}$ instead of $\varphi$, with $E$ and $E^{\prime}, W$ and $W^{\prime}, S$ and $S^{\prime}$ interchanged, we find that $\sigma^{-1}$ is also continuous, and so $\sigma$ is a homeomorphism.

The mapping $\Gamma: P\left(W^{\prime}\right) \times \boldsymbol{P}^{\times} \rightarrow P\left(W^{\prime}\right) \times \boldsymbol{P}^{\times}$defined by $(6.2)$ is invertible, and its inverse is given by

$$
\Gamma^{-1}\left(p^{\prime}, \alpha\right)=\left(p^{\prime},\left(\gamma_{p^{\prime}}\right)^{-1}(\alpha)\right) \quad \text { for all }\left(p^{\prime}, \alpha\right) \in P\left(W^{\prime}\right) \times \boldsymbol{P}^{\times} \text {. }
$$

If $P\left(W^{\prime}\right)=\emptyset, \Gamma$ and $\Gamma^{-1}$ are trivially continuous. We therefore assume that $P\left(W^{\prime}\right) \neq \emptyset$ and choose $q^{\prime} \in P\left(W^{\prime}\right)$. Then (6.4) and (4.1) imply

$$
\begin{aligned}
& \gamma_{p^{\prime}}=\mathrm{ev}_{p^{\prime}}^{s^{\prime}} \circ\left(\left.\varphi\right|_{S} ^{\mid s^{\prime}}\right) \circ\left(\mathrm{ev}_{\sigma^{\prime}\left(p^{\prime}\right)}^{s}\right)^{-1}=
\end{aligned}
$$

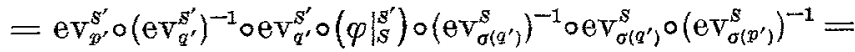

$$
\begin{aligned}
& =\omega_{S^{\prime}}\left(q^{\prime}, p^{\prime}, \cdot\right) \circ \gamma_{q^{\prime}} \circ \omega_{\mathcal{S}}\left(\sigma\left(p^{\prime}\right), \sigma\left(q^{\prime}\right), \cdot\right) \quad \text { for all } p^{\prime} \in P\left(W^{\prime}\right),
\end{aligned}
$$

and similarly

$$
\left(\gamma_{r^{\prime}}\right)^{-1}=\omega_{S}\left(\sigma\left(q^{\prime}\right), \sigma\left(p^{\prime}\right), \cdot\right) \circ\left(\gamma_{q^{\prime}}\right)^{-1} \circ \omega_{S^{\prime}}\left(p^{\prime}, q^{\prime}, \cdot\right) \quad \text { for all } p^{\prime} \in P\left(W^{\prime}\right)
$$

Now we have shown that $\sigma$ is continuous; $\gamma_{q^{\prime}}$ and $\left(\gamma_{q^{\prime}}\right)^{-1}$ are continuous, since they are $<-<$-isomorphisms from $\boldsymbol{P} \times$ to $\boldsymbol{P}^{\times}$; and $\omega_{s}$ and $\omega_{S^{\prime}}$ are continuous by Proposition 4.3. It follows from $(6.2),(6.6),(6.7),(6.8)$ that $\Gamma$ and $\Gamma^{-1}$ are also continuous.

For given $E$ and $E^{\prime}, W$ and $W^{\prime}$, the significance of Theorem 6.1 depends, of course, on how large $P(W)$ and $P\left(W^{\prime}\right)$ are in $E$ and $E^{\prime}$, respectively. In particular, this theo- 
rem gives a description of a sealed $\leqslant-\leqslant$-isomorphism when either $P(W)=E$ or $P\left(W^{\prime}\right)=E^{\prime}$. When both equalities hold, we can show that a scaled $\leqslant-\leqslant$-isomorphism must be a $<-<$-isomorphism. For a slightly better answer see Proposition 7.4.

6.2. Corollary. - If $P(W)=E$ and $P\left(W^{\prime}\right)=E^{\prime}$ and there exists a scaled $\leqslant-\leqslant-i s o-$ morphism from $W^{+}$to $W^{\prime+}$, then $E$ and $E^{\prime}$ are homeomorphic, and every scaled $\leqslant-\leqslant-i s o-$ morphism from $W^{+}$to $W^{\prime+}$ is a $<-<-$ isomorphism.

Proof. - Let $\varphi: W^{+} \rightarrow W^{\prime+}$ be a scaled $\leqslant-\leqslant$-isomorphism, and let $\sigma$ and $\left(\gamma_{g^{\prime}}\right)$ $\left.p^{\prime} \in E^{\prime}\right)$ be as in Theorem 6.1. Then $\sigma: E^{\prime} \rightarrow E$ is a homeomorphism. Let $f, g \in W^{+}$ be given. By (6.1) we have the following chain of equivalences for every $p^{\prime} \in E^{\prime}$ :

$$
\begin{aligned}
f\left(\sigma\left(p^{\prime}\right)\right)<g\left(\sigma\left(p^{\prime}\right)\right) & \Leftrightarrow \gamma_{p^{\prime}}\left(f\left(\sigma\left(p^{\prime}\right)\right)\right)<\gamma_{p^{\prime}}\left(g\left(\sigma\left(p^{\prime}\right)\right)\right) \Leftrightarrow \\
& \Leftrightarrow(\varphi(f))\left(p^{\prime}\right)<(\varphi(g))\left(p^{\prime}\right) .
\end{aligned}
$$

Since $\sigma: E^{\prime} \rightarrow E$ is surjective, this shows that $f<g$ if and only if $\varphi(f)<\varphi(g)$.

6.3. ExanPLES. - We refer to Examples 2.2. If $W:=\operatorname{Cont}(E)$ or $W:=\operatorname{Anal}(E)$ or $W:=\operatorname{Pol}(E)$ (with $E$ as described there in each case), then $P(W)=E$. For the first example, this is an instance of the criterion given in Proposition 6.4 below. For the second and third examples, this is shown by choosing a euclidean metric $d$ on the affine space $X$ and remarking that for each given $p \in E$ the function $f: E \rightarrow \boldsymbol{R}$ defined by $f(q):=d(p, q)^{2}$ for all $q \in E$ satisfies $f \in \overline{\mathrm{Pol}}^{+}(E) \subset \overline{\mathrm{Anal}}+(E)$ and $f(p)=$ $=0<f(q)$ for all $q \in E \backslash\{p\}$.

On the other hand, one ean show that if $E$ is the compact convex set without "algebraically exposed point " described in [2; Section 2], then $P($ Aff $(E))=\emptyset$. This example is due to R. R. PHelps (private communication).

The following result gives a somewhat special condition for $P(W)=E$.

6.4. Proposition. - If $W$ is a sublattice of Cont $(E)$, then $P(W)=E$.

Proof. - Let $p, q \in E$ be given, with $p \neq q$. By $\left(\mathrm{W}_{2}\right)$ we may choose $w \in W$ such that $w(p)=0 \neq w(q)$, and we may assume without loss that $w(q)>0$. Then $f:=\sup \{0, w\} \in W \cap{\overline{\text { Cont }^{+}}}^{+}(E)=\bar{W}$ satisfies $f(p)=0<f(q)$. Hence $p \in P(W)$ for every $p \in E$.

In the special case in which $W:=\operatorname{Cont}(E)$ and $W_{1}:=\operatorname{Cont}\left(E^{\prime}\right)$, the representation given by Theorem 6.1 of all $<-<$-isomorphisms from $\mathrm{Cont}^{+}(E)$ to $\mathrm{Cont}^{+}\left(E^{\prime}\right)$ is a complete description of them, as we now show.

6.5. THEORER. - (a) Assume that $\varphi$ : Cont ${ }^{+}(E) \rightarrow$ Cont $^{+}\left(E^{\prime}\right)$ is a $<-<-$ isomorphism (equivalently, a scaled $\leqslant-\leqslant-$-isomorphism). Then there exists a unique combination 
of a bijection $\sigma: E^{\prime} \rightarrow E$ and a family $\left(\gamma_{v} \mid p^{\prime} \in E^{\prime}\right)$ of $<-<-$-isomorphisms from $\boldsymbol{P}^{\times}$to $\boldsymbol{P}^{\times}$such that

$$
(\varphi(f))\left(p^{\prime}\right)=\gamma_{p^{\prime}}\left(f\left(\sigma\left(p^{\prime}\right)\right)\right) \quad \text { for all } f \in \operatorname{Cont}^{+}(E) \text { and } p^{\prime} \in E^{\prime}
$$

Moreover, $\sigma$ and the mapping $\Gamma: E^{\prime} \times \boldsymbol{P}^{\times} \rightarrow E^{\prime} \times \boldsymbol{P}^{\times}$defined by

$$
\Gamma\left(p^{\prime}, \alpha\right):=\left(p^{\prime}, \gamma_{p^{\prime}}(\alpha)\right) \quad \text { for all }\left(p^{\prime}, \alpha\right) \in E^{\prime} \times \boldsymbol{P}^{\times}
$$

are homeomorphisms.

(b) If $\sigma: E^{\prime} \rightarrow E$ is a homeomorphism and $\left(\gamma_{p^{\prime}} \mid p^{\prime} \in E^{\prime}\right)$ is a family of $<-<-i s o-$ morphisms from $\boldsymbol{P}^{\times}$to $\boldsymbol{P}^{\times}$such that the mapping $\Gamma: E^{\prime} \times \boldsymbol{P}^{\times} \rightarrow E^{\prime} \times \boldsymbol{P}^{\times}$defined by (6.11) is a homeomorphism, then (6.10) defines $a<-<-$ isomorphism $\varphi$ : Cont $^{+}(E) \rightarrow$ Cont $^{+}\left(E^{\prime}\right)$.

Proof, - Part (a) is an immediate consequence of Theorem 6.1, Corollaries 5.2 and 6.2, and Proposition 6.4.

In order to prove Part $(b)$, let $\sigma$ and $\left(\gamma_{p^{\prime}} \mid p^{\prime} \in E^{\prime}\right)$ be given and let the assumptions be satisfied. For every $f \in \operatorname{Cont}^{+}(E)$ a function $\varphi(f): E^{\prime} \rightarrow \boldsymbol{R}$ is defined by (6.10), and for every $f^{\prime} \in \operatorname{Cont}^{+}\left(E^{\prime}\right)$ a function $\varphi^{\prime}\left(f^{\prime}\right): E \rightarrow \boldsymbol{R}$ is defined by

$$
\left(\varphi^{\prime}\left(f^{\prime}\right)\right)(p):=\left(\gamma_{\sigma^{-1}(p)}\right)^{-1}\left(f^{\prime}\left(\sigma^{-1}(p)\right)\right) \text { for all } p \in E .
$$

It follows from these definitions and from (6.11) that

$$
\begin{aligned}
& \left(p^{\prime},(\varphi(f))\left(p^{\prime}\right)\right)=\Gamma\left(p^{r}, f\left(\sigma\left(p^{\prime}\right)\right)\right) \quad \text { for all } f \in \operatorname{Cont}^{+}(E) \text { and } p^{\prime} \in E^{\prime} . \\
& \left(\sigma^{-1}(p),\left(\varphi^{\prime}\left(f^{\prime}\right)\right)(p)\right)=\Gamma^{-1}\left(\sigma^{-1}(p), f^{\prime}\left(\sigma^{-1}(p)\right)\right) \\
& \text { for all } f^{\prime} \in \operatorname{Cont}^{+}\left(E^{\prime}\right) \text { and } p \in E .
\end{aligned}
$$

Since $\sigma$ and $\Gamma$ are homeomorphisms, it follows from (6.13) and (6.14) that $\varphi(f)$ and $\varphi^{\prime}\left(f^{\prime}\right)$ are continuous and strictly-positive-valued for all $f \in \operatorname{Cont}^{+}(E)$ and $f^{\prime} \in \mathrm{Cont}^{+}\left(E^{\prime}\right)$. Thus (6.10) and (6.12) define mappings $\varphi$ : Cont ${ }^{+}(E) \rightarrow \operatorname{Cont}^{+}\left(E^{\prime}\right)$ and $\varphi^{\prime}: \mathrm{Cont}^{+}\left(E^{\prime}\right) \rightarrow$ $\rightarrow$ Cont $^{+}(E)$; and it is immediately verified that these mappings are inverse to each other. Thus $\varphi$ is invertible.

Let $f, g \in \operatorname{Cont}^{+}(E)$ be given. For each $p^{\prime} \in E^{\prime}$ we have the chain (6.9) of equivalences. Thus $f<g$ if and only if $\varphi(f)<\varphi(g)$. Hence $\varphi$ is a $<-<$-isomorphism.

REMARK. - Kaplansky's Theorem ([5]) implies that the existence of a $\leqslant-\leqslant-$ isomorphism from $\operatorname{Cont}^{+}(E)$ to $\operatorname{Cont}^{+}\left(E^{\prime}\right)$ forces $E$ and $E^{\prime}$ to be homeomorphic. On comparing this result with Theorem 6.5 , one might be tempted to conjecture that, in the case of the cones $\operatorname{Cont}^{+}(E)$ and $\operatorname{Cont}^{+}\left(E^{\prime}\right)$ at least, every $\leqslant-\leqslant$-isomorphism must be a $<-<$-isomorphism. However, NANIOKA and SAEKI $[9$; Theorems 1.5 and $2.10]$ have recently shown that this implication fails when-indeed, precisely when- $E$, 
and hence $E^{\prime}$, is homeomorphic to the Stone-Čech compactification of a non-compact $\sigma$-compact locally eompact Hausdorff space. (Added in proof).

6.6. Examples. - We have nothing comparable to Theorem 6.5 with regard to necessary and sufficient conditions on $\sigma$ and $\left(\gamma_{p^{\prime}} \mid p^{\prime} \in E^{\prime}\right)$ for our other examples with $P(W)=E$ and $P\left(W^{\prime}\right)=E^{\prime}$ (Examples 6.3). We point out that, for each space Anal $(E)$ and each $\alpha \in \boldsymbol{P}^{\times}$, the valuewise power mapping $f \mapsto f^{\alpha}:$ Anal $^{+}(E) \rightarrow$ Anal $^{+}(E)$ is a $<-<$-isomorphism, but is not a cone-isomorphism unless $\alpha=1$. On the other hand, we-conjecture that every <-<-isomorphism $\varphi: \mathrm{Pol}^{+}(E) \rightarrow \mathrm{Pol}^{+}(E)$ is a coneisomorphism (and hence of the form described in Examples 8.5).

\section{7. - Some refinements.}

One defect of the results in the preceding two sections lies in the fact that, at best, they provide information on the values of the functions under consideration only at the points of the sets $Z \in \mathcal{Z}(W)$ and $Z^{I} \in \mathcal{Z}\left(W^{\prime}\right)$. We now give a few results that contribute to remedy this defect at least in part.

In this section, we assume that $E$ is a non-empty compact Hausdorff space and that $W$ is a subspace of Cont $(E)$ that satisfies $\left(\mathrm{W}_{1}\right)$ and $\left(\mathrm{W}_{2}\right)$.

7.1. Propositron. - For every $f \in \overline{W^{+}} \backslash W^{+}$there exists $Z \in \mathfrak{3}(W)$ such that $f_{>}(Z)=$ $=\{0\}$. In particular, $\tilde{3}(W) \neq \emptyset$.

Proof. - Every singleton in $\bar{W}^{+} \backslash W^{+}$is a $\leqslant$-directed subset, and the union of each collection of $\leqslant$-directed subsets of $\overline{W^{+}} \backslash W^{+}$that is totally ordered by inclusion is clearly $a \leqslant$-directed subset of $\bar{W}^{+} \backslash W^{+}$. By Zorn's Lemma, $\bar{W}^{+} \backslash W^{+}$is the union of the collection of its maximal $\leqslant$-directed subsets. By Proposition 3.2, this implies

$$
\bar{W}+\backslash W^{+}=\bigcup\left\{J_{W F}(Z) \mid Z \in \mathfrak{Z}(W)\right\},
$$

and the assertion follows at once.

7.2. Proposition. - Let $E_{0}$ be a compact subset of $E$ such that $E_{0} \cap Z \neq \emptyset$ for all $Z \in \mathcal{Z}(W)$. Let $W_{0}$ be the subspace of Cont $\left(E_{0}\right)$ consisting of the restrictions to $E_{0}$ of all elements of $W$. Then $W_{0}$ satisfies $\left(W_{1}\right)$ and $\left(W_{2}\right)$; the mapping $\varrho: W \rightarrow W_{0}$ defined $b y \varrho(f):=\left.f\right|_{E_{0}}$ for all $f \in W$ is a linear isomorphism and has a restriction to a coneisomorphism from $W^{+}$to $W_{0}^{+}$; and

$$
\mathcal{Z}\left(W_{0}\right)=\left\{E_{0} \cap Z \mid Z \in \mathcal{Z}(W)\right\} .
$$

Proof. $-E_{0} \neq \emptyset$ by Proposition 7.1. The facts that $W_{0}$ satisfies $\left(W_{1}\right)$ and $\left(W_{2}\right)$ and that $\varrho$ is linear and surjective are trivial consequences of the definitions. It is equally obvious that

$$
\varrho_{>}\left(\bar{W}^{+}\right) \subset \bar{W}_{0}^{+} .
$$


We choose a fixed $w \in W^{+}$. Let $f_{0} \in \bar{W}_{0}^{+}$be given, and let $f \in W$ be such that $f_{0}=\varrho(f)=\left.f\right|_{E_{0}}$ : Then $f-(\min (f / w)) w \in \bar{W}^{+} \backslash W^{+}$. By 'Proposition 7.1 we may choose $Z \in 3(W)$ such that $(f-(\min (f / w)) w)>(Z)=\{0\}$; by the assumption we may choose $p \in E_{0} \cap Z$, and find

$$
(\min (f / w)) w(p)=f(p)=f_{0}(p) \geqslant 0,
$$

so that $\min (f / w) \geqslant 0$, and hence $f \in \bar{W}^{+}$. We have shown that

$$
\varrho^{<}\left(\bar{W}_{0}^{+}\right) \subset \bar{W}^{+} .
$$

If $f \in W$ satisfies $\varrho(f)=0 \in \bar{W}_{0}^{+} \cap\left(-\bar{W}_{0}^{+}\right),(7.3)$ implies that $f \in \varrho^{<}\left(\bar{W}_{0}^{+} \cap\left(-\bar{W}_{0}^{+}\right)\right) c$ $\subset \bar{W}^{+} \cap\left(-\bar{W}^{+}\right)=\{0\}$, so that $f=0$. Thus $\varrho$ is injective, and hence a linear isomorphism, as asserted. (7.2) and (7.3) then show that

$$
\varrho_{>}\left(\bar{W}^{+}\right)=\bar{W}_{0}^{+} .
$$

Since $W^{+}=\boldsymbol{P}^{\times} w+\bar{W}^{+}$and $W_{0}^{+}=\boldsymbol{P}^{\times} \varrho(w)+\bar{W}_{0}^{+}$, it follows from (7.4) that

$$
\varrho_{>}\left(W^{+}\right)=W_{0}^{+}
$$

and therefore the linear isomorphism $\varrho$ has a restriction to a cone-isomorphism from $W^{+}$ to $W_{0}^{+}$.

In view of (7.4) and (7.5), $\varrho$ has a restriction to a $\leqslant-\leqslant$-isomorphism from $\overline{W^{+} \backslash W^{+}}$ to $\bar{W}_{0}^{+} \backslash W_{0}^{+}$. Hence the mapping $\varrho_{>}: \mathfrak{B}(W) \rightarrow \mathfrak{B}\left(W_{0}\right)$ has a restriction to a bijection from the collection of all maximal $\leqslant$-directed subsets of $\bar{W} \backslash \backslash W^{+}$to the collection of all maximal $\leqslant$-directed subsets of $\bar{W}_{0}^{+} \backslash W_{0}^{+}$. By Proposition 3.2 it follows that there is a bijection $\Phi: \mathfrak{Z}(w) \rightarrow \mathfrak{Z}\left(W_{0}\right)$ such that

$$
J_{W_{0}}(\Phi(Z))=\varrho_{>}\left(J_{W}(Z)\right) \quad \text { for all } Z \in \mathcal{Z}(W) .
$$

But them

$$
\begin{aligned}
\Phi(Z) & =\bigcap\left\{\left(f_{0}\right)<(\{0\}) \mid f_{0} \in J_{W_{0}}(\Phi(Z))\right\}=\bigcap\left\{E_{0} \cap f^{<}(\{0\}) \mid f \in J_{W}(Z)\right\}= \\
& =E_{0} \cap \bigcap\left\{f^{<}(\{0\}) \mid f \in J_{W}(Z)\right\}=E_{0} \cap Z
\end{aligned}
$$

for all $Z \in 3(W)$. Since $\Phi$ is a bijection, (7.1) follows.

7.3. COROLLARY. - Let $E_{W}$ be the closure of $\bigcup \mathcal{Z}(W)$, and let $W_{0}$ be the subspace of Cont $\left(E_{W}\right)$ consisting of the restrictions to $E_{W}$ of all elements of $W$. Then $W_{0}$ satisfies $\left(W_{1}\right)$ and $\left(W_{2}\right)$; the mapping $\varrho_{W}: W \rightarrow W_{0}$ defined by $\varrho_{W}(f):=\left.f\right|_{E_{W}}$ for all $f \in W$ is a linear isomorphism and has a restriction to a cone-isomorphism $\psi_{w}: W^{+} \rightarrow W_{0}^{+} ;$and $3\left(W_{0}\right)=$ $=3(W)$. 
REMARK. - It is a consequence of Corollary 7.3 (with Lemma 3.7) that an isolated $Z \in \xi(W)$, i.e., one that is disjoint from the closure of $U(\xi(W) \backslash\{Z\})$, must be a singleton.

Proposition 7.2 and Corollary 7.3 may be used in conjunction with Theorem 6.1 in order to broaden the range of applicability of the latter. We shall use them in this section and the next to obtain certain more generic results, and we begin by deriving a slightly strengthened version of part of Corollary 6.2.

7.4. Proposttion. - Let $E$ and $E^{\prime}, W$ and $W^{\prime}$ be as in Section 6. Assume that $P(W)=\bigcup \mathfrak{Z}(W)$ and $P\left(W^{\prime}\right)=\bigcup \mathfrak{Z}\left(W^{\prime}\right)$ (i.e., every element of $\mathfrak{Z}(W)$ and of $\mathfrak{3}\left(W^{\prime}\right)$ is a singleton) and that both these sets are closed. Then every scaled $\leqslant-\leqslant-$ isomorphism from $W^{+}$to $W^{\prime+}$ is necessarily a $<-<$-isomorphism.

Proof. - Let $E_{W}, W_{0}, \psi_{W}$ be as in Corollary 7.3, and $E_{W^{\prime}}^{\prime}, W_{0}^{\prime}, \psi_{W^{\prime}}$ corresponding objects for $E^{\prime}$ and $W^{\prime}$. Then that corollary and the assumption yield $P\left(W_{0}\right)=$ $=P(W)=E_{W}$ and $P\left(W_{0}^{\prime}\right)=P\left(W^{\prime}\right)=E_{W}^{\prime}:$ Let $\varphi: W^{+} \rightarrow W^{\prime+}$ be a scaled $\leqslant-\leqslant-$ isomorphism. Since $\psi_{W}$ and $\psi_{W^{\prime}}$, are cone-isomorphisms, they are $<-<$-isomorphisms and hence also, by Corollary $5.2, \leqslant-\leqslant$-isomorphisms scaled by every $W$-scale or $W^{\prime}$-scale, respectively. Therefore $\psi_{W^{\prime}} \circ \circ \circ\left(y^{\prime} w\right)^{-1}: W_{0}^{+} \rightarrow W_{0}^{\prime+}$ is a scaled $\leqslant-\leqslant$-isomorphism. By Corollary 6.2, this is necessarily a $<-<$-isomorphism. Hence $\varphi$ is also a $<-<$-isomorphism.

We next examine the consequences of assuming that $\leqslant$ is a lattice-order on $W$. The first proposition, which is preliminary to that examination, may prove of interest in specifying conditions under which the order $\leqslant$ is loose, in the sense of [7; Section 9] (an order is ioose, roughly speaking, if a doubleton has an infimum or a supremum only when one element precedes the other).

7.5. Proposition. - Let $E_{W}$ be the closure of $\bigcup 3(W)$. Let $f, g \in W$ be given. Then 0 is the $\leqslant-$ infimum in $W$ of $\{f, g\}$ (i.e., $\left.\left(f-\bar{W}^{+}\right) \cap\left(g-\bar{W}^{+}\right)=-\bar{W}^{+}\right)$if and only if $0=\inf \left\{\left.f\right|_{E_{w}},\left.g\right|_{E_{w}}\right\}$.

Proof. - Let $E_{0}$ and $\varrho_{W}$ be as in Corollary 7.3, and set $f_{0}:=\left.f\right|_{E_{W}}=\varrho_{W}(f), g_{0}:=$ $:=\left.g\right|_{E_{W}}=\varrho_{W}(g)$. By that corollary, $\left(f-\bar{W}^{+}\right) \cap\left(g-\bar{W}^{+}\right)=-\bar{W}^{+}$if and only if

$$
\left(f_{0}-\bar{W}_{0}^{+}\right) \cap\left(g_{0}-\bar{W}_{0}^{+}\right)=-\bar{W}_{0}^{+} .
$$

If $0=\inf \left\{f_{0}, g_{0}\right\}$, we have

$$
\left(f_{0}-\overline{\operatorname{Cont}}^{+}\left(E_{W}\right)\right) \cap\left(g_{0}-{\overline{\operatorname{Cont}^{+}}}^{+}\left(E_{W}\right)\right)=-\overline{\operatorname{Cont}}^{+}\left(E_{W}\right),
$$

and (7.6) follows upon intersecting both sides of this equation with $W_{0}$.

Assume, conversely, that (7.6) holds. Then $f_{0}, g_{0} \in \bar{W}_{0}^{+} \subset \overline{\text { Cont }}^{+}\left(E_{W}\right)$, so that $\inf \left\{f_{0}, g_{0}\right\} \geqslant 0$. Suppose that $\inf \left\{f_{0}, g_{0}\right\} \neq 0$; since this value-wise infimum is a con- 
tinuous function, we may choose $p \in \bigcup \zeta(W)$ such that $f_{0}(p)>0$ and $g_{0}(p)>0$, and find the unique $Z \in Z(W)$ such that $p \in Z$. By Corollary 7.3, $Z \in Z\left(W_{0}\right)$. It follows from $\left(Z_{1}\right)$ that $\left.f_{0}\right|_{z}>0,\left.g_{0}\right|_{z}>0$.

We choose a fixed $w_{0} \in W_{0}^{+}$. We may then choose $\alpha \in \boldsymbol{P}^{\times}$so little that $\left.f_{0}\right|_{z}>\left.\alpha w_{0}\right|_{z}$, $\left.g_{0}\right|_{z}>\left.\alpha w_{0}\right|_{z}$. By Proposition 3.6 we may further choose $h_{0}, k_{0} \in J_{W_{0}}(Z)$ such that $\alpha w_{0}<f_{0}+h_{0}, \alpha w_{0}<g_{0}+k_{0}$. Then $\alpha w_{0}-h_{0}-k_{0} \in\left(f_{0}-\bar{W}_{0}^{+}\right) \cap\left(g_{0}-\bar{W}_{0}^{+}\right)$, but $\left(\alpha w_{0}-\right.$ $\left.-h_{0}-h_{0}\right)(p)=\alpha w_{0}(p)>0$, so that $\alpha w_{0}-h_{0}-h_{0} \notin-\bar{W}_{0}^{+}$, contradicting (7.6). Our supposition that $\inf \left\{f_{0}, g_{0}\right\} \neq 0$ is thus untenable.

7.6. Corollary. - Let $E_{W}$ be the closure of $\bigcup 3(W)$ and $W_{0}$ the subspace of Cont $\left(E_{W}\right)$ consisting of the restrictions to $E_{W}$ of all elements of $W$. If $\leqslant$ is a lattice-order on $W$, then $W_{0}$ is a sublattice of Cont $\left(E_{W}\right)$.

7.7. ExAMplat. - If $\leqslant$ is a lattice-order on $W, W$ need of course not be a sublattice of Cont $(E)$. For instance, if $E:=[0,1], W:=$ Aff $([0,1])$, we have $3(W)=$ $=\{\{0\},\{1\}\}$ and $P(W)=\bigcup \mathfrak{Z}(W)=\{0,1\}$. Aff $([0,1])$ is lattice-ordered by $\leqslant$, but is of course not a sublattice of Cont $([0,1])$.

7.8. Proposition, $-I f \leqslant$ is a lattice-order on $W$, then $P(W)=\bigcup \mathfrak{Z}(W)$, and this set is closed.

Proof. - Let $E_{W}$ and $W_{0}$ be as in Corollaries 7.3 and 7.6. By Corollary 7.6, $W_{0}$ is a sublattice of Cont $\left(E_{W}\right)$. By Proposition 6.4, $P\left(W_{0}\right)=E_{W}$ : By Corollary 7.3, $P\left(W_{0}\right)=P(W) \subset \bigcup \mathfrak{Z}(W)$. Since $E_{W}$ is the closure of $\bigcup \mathfrak{Z}(W)$, the conclusion follows.

\section{8. - Order-isomorphisms with a homogeneity condition.}

As in Section 6, we assume that $E$ and $E^{\prime}$ are non-empty compact Hausdorff spaces, and that $W$ and $W^{\prime}$ are subspaces of Cont $(E)$ and Cont $\left(E^{\prime}\right)$, respeetively, and that both satisfy $\left(\mathrm{W}_{1}\right)$ and $\left(\mathrm{W}_{2}\right)$.

As already suggested in the introduction, we are interested in finding out when given $\leqslant-\leqslant$-isomorphism $\varphi: W^{+} \rightarrow W^{\prime+}$ is in fact a cone-isomorphism. This will not in general follow without additional conditions (see Theorem 6.5 and Examples 6.6), although it does for certain spaces $W$ and $W^{\prime}$ (for an abstract condition of this kind, see [7; Theorem A and Corollary A1]). A necessary condition is that $\varphi$ be homogeneous, i.e., that it satisfy $\varphi(\alpha f)=\alpha \varphi(f)$ for all $\alpha \in \boldsymbol{P}^{\times}$and all $f \in W^{+}$. It turns out that this condition is also sufficient; this is shown in [8; Theorem B] (in conjunction with [7; Proposition 7.2]), using the results obtained in this section. We shall, in fact, consider here a weaker condition, namely that $\varphi$ is homogeneous at $w$ for some $w \in W^{+}$, which means that $\varphi(\alpha f)=\alpha \varphi(f)$ for all $\alpha \in \boldsymbol{P}^{\times}$. 
8.1. TheoRem. - Assume that $\varphi: W^{+} \rightarrow W^{\prime+}$ is a $\leqslant-\leqslant$-isomorphism and that is is homogeneous at a given $w \in W^{+}$. Then there exists a unique bijection $\sigma: P\left(W^{\prime}\right) \rightarrow P(W)$ such that

$$
(\varphi(f))\left(p^{\prime}\right)=\frac{(\varphi(w))\left(p^{\prime}\right)}{w\left(\sigma\left(p^{\prime}\right)\right)} f\left(\sigma\left(p^{\prime}\right)\right) \quad \text { for all } f \in W^{+} \text {and } p^{\prime} \in P\left(W^{\prime}\right)
$$

moreover, $\sigma$ is a homeomorphism.

Proof. $-\boldsymbol{P}^{\times} w$ is a $W$-scale and, by the homogeneity assumption, $\varphi_{>}(\boldsymbol{P} \times w)=$ $=\boldsymbol{P}^{\times} \varphi(w)$ is a $W^{\prime}$-scale. Thus $\varphi$ is scaled and Theorem 6.1 is applicable. With $\sigma$ and $\left(\gamma_{p^{\prime}} \mid p^{\prime} \in P\left(W^{\prime}\right)\right)$ as given by that theorem, we find that $\sigma$ is a homeomorphism, an application of $(6.1)$ to $f:=w$ yields

$$
\begin{aligned}
\gamma_{p^{\prime}}\left(\alpha w\left(\sigma\left(p^{\prime}\right)\right)\right) & =\gamma_{p^{\prime}}\left((\alpha w)\left(\sigma\left(p^{\prime}\right)\right)\right)=(\varphi(\alpha w))\left(p^{\prime}\right)=(\alpha \varphi(w))\left(p^{\prime}\right)= \\
& =\alpha(\varphi(w))\left(p^{\prime}\right) \quad \text { for all } p^{\prime} \in P\left(W^{\prime}\right) \text { and } \alpha \in \boldsymbol{P}^{\times},
\end{aligned}
$$

so that

$$
\gamma_{p^{\prime}}(\alpha)=\frac{(\varphi(w))\left(p^{\prime}\right)}{w\left(\sigma\left(p^{\prime}\right)\right)} \alpha \quad \text { for all } p^{\prime} \in P\left(W^{\prime}\right) \text { and } \alpha \in \boldsymbol{P}^{\times}
$$

Combining (8.2) with (6.1) we obtain (8.1).

Conversely, if $\sigma: P\left(W^{\prime}\right) \rightarrow P(W)$ is a bijection such that (8.1) holds, it follows that (6.1) holds with that same $\sigma$ and with $\left(\gamma_{p^{\prime}} \mid p^{\prime} \in P\left(W^{\prime}\right)\right)$ defined by (8.2) (this does define a family of $<-<$-isomorphisms from $\boldsymbol{P} \times$ to $\left.\boldsymbol{P}^{\times}\right)$. The present uniqueness assertion then follows from the uniqueness assertion of Theorem 6.1,(a).

8.2. Corollary. - Assume that $P(W)$ is dense in $\bigcup \mathfrak{z}(W)$ or $P\left(W^{\prime}\right)$ is dense in $\bigcup \mathfrak{Z}\left(W^{\prime}\right) . \quad A \leqslant-\leqslant-$ isomorphism $\varphi: W^{+} \rightarrow W^{\prime+}$ that is homogeneous at some $w \in W^{+}$ is then necessarily a cone-isomorphism.

Proof. - If $\varphi$ is homogeneous at $w$, then $\varphi^{-1}$ is homogeneous at $\varphi(w)$. It is therefore sufficient to prove the assertion under the assumption that $P\left(W^{\prime}\right)$ is dense in $\bigcup \tilde{\mathfrak{Z}}\left(W^{\prime}\right)$.

Let $E_{W^{\prime}}^{\prime}, W_{\mathbf{0}}^{\prime}, \psi_{W^{\prime}}$, be as in Corollary 7.3, with $E^{\prime}$ and $W^{\prime}$ instead of $E$ and $W$. For all $f, g \in W^{+}$and $\alpha, \beta \in \boldsymbol{P}^{\times}$we have, by Theorem 8.1,

$$
\begin{aligned}
(p(\alpha f+\beta g))\left(p^{\prime}\right) & =\frac{(\varphi(w))\left(p^{\prime}\right)}{w\left(\sigma\left(p^{\prime}\right)\right)}(\alpha f+\beta g)\left(\sigma\left(p^{\prime}\right)\right)= \\
& =\alpha \frac{(\varphi(w))\left(p^{\prime}\right)}{w\left(\sigma\left(p^{\prime}\right)\right)} f\left(\sigma\left(p^{\prime}\right)\right)+\beta \frac{(\varphi(w))\left(p^{\prime}\right)}{w\left(\sigma\left(p^{\prime}\right)\right)} g\left(\sigma\left(p^{\prime}\right)\right)= \\
& =\alpha(\varphi(f))\left(p^{\prime}\right)+\beta(\varphi(g))\left(p^{\prime}\right)=(\alpha \varphi(f)+\beta \varphi(g))\left(p^{\prime}\right)
\end{aligned}
$$

for all $p^{\prime} \in P\left(W^{\prime}\right)$. Thus the continuous functions $\varphi(\alpha f+\beta g)$ and $\alpha \varphi(f)+\beta \varphi(g)$ 
agree on $P\left(W^{\prime}\right)$, which is dense in $\bigcup Z\left(W^{\prime}\right)$ and hence in the closure $E_{W}^{\prime}$, of the latter set. Therefore they agree on $E_{W^{\prime}}^{\prime}$, so that

$\left(\psi_{W^{\prime}} \circ \varphi\right)(\alpha f+\beta g)=\left.\varphi(\alpha f+\beta g)\right|_{E_{W^{\prime}}^{\prime}}=\left.\alpha \varphi(f)\right|_{E_{W^{\prime}}^{\prime}}+\left.\beta \varphi(g)\right|_{E_{W^{\prime}}^{\prime}}=\alpha\left(\psi_{W^{\prime}} \circ \varphi\right)(f)+\beta\left(\psi_{W^{\prime}} \circ \varphi\right)(g)$.

We conclude that $\psi_{W^{\prime}} \circ \varphi: W^{+} \rightarrow W_{0}^{\prime+}$ is linear; since $\psi_{W^{\prime}}$ is a cone-isomorphism, $\varphi=\left(\psi_{W^{\prime}}\right)^{-1} \circ\left(\psi_{W^{\prime}} \circ \varphi\right)$ is also linear; since it is bijective, it is a cone-isomorphism.

8.3. CoRollary. - Assume that $\leqslant$ is a lattice-order on $W$ or that $\leqslant$ is a latticeorder on $W^{\prime} . A \leqslant-\leqslant-i s o m o r p h i s m ~ \varphi: W^{+} \rightarrow W^{+}$that is homogeneous at some $w \in W^{+}$ is then necessarily a cone-isomorphism.

Pkoof. - Corollary 8.2 and Proposition 7.8.

8.4. Corollary. - Assume that $P(W)=E$ and $P\left(W^{\prime}\right)=E^{\prime}$. If $\varphi: W^{+} \rightarrow W^{\prime+}$ is $a \leqslant-\leqslant-i$ somorphism that is homogeneous at some $w \in W^{+}$, then $\varphi$ is a cone-isomorphism and there exists a unique combination of a homeomorphism $\sigma: E^{\prime} \rightarrow E$ and a function $h \in$ Cont $^{+}\left(E^{\prime}\right)$ such that

$$
\varphi(f)=h \cdot(f \circ \sigma) \quad \text { for all } f \in W^{+} .
$$

Proof. - Theorem 8.1 yields (8.3) with a homeomorphism $\sigma$ and with $h:=\varphi(w) /(w \circ \sigma) ; \varphi$ is thus of course a cone-isomorphism (cf. Corollary 8.2). Conversely, (8.3) applied to $f:=w$ yields $h=\varphi(w) /(w \circ \sigma)$; so that the present uniqueness assertion follows from the uniqueness assertion of Theorem 8.1.

Ramark. - By virtue of the discussion in Examples 6.3, this corollary provides a proof of $[7$; Theorem 8].

8.5. Examples. - Corollary 8.4 is applicable when $W:=\operatorname{Cont}(E)$ and $W:=$ $:=$ Cont $\left(E^{\prime}\right)$ (cf. Examples 6.3). In this case, every combination of a homeomorphism $\sigma: E^{\prime} \rightarrow E$ and a function $h \in \operatorname{Cont}^{+}\left(E^{\prime}\right)$ defines, by (8.3), a cone-isomorphism $\varphi$ : $\operatorname{Cont}^{+}(E) \rightarrow$ Cont $^{+}\left(E^{\prime}\right)$.

For other combinations of $W$ and $W^{\prime}$ with $P(W)=E$ and $P\left(W^{\prime}\right)=E^{\prime}$, the combinations of homeomorphism $\sigma: E^{\prime} \rightarrow E$ and $h \in \mathrm{Cont}^{+}\left(E^{\prime}\right)$ that define, by (8.3), a cone-isomorphism $p: W^{+} \rightarrow W^{\prime}$ are of course subject to further restrictions.

Thus, if $E$ and $E^{\prime}$ are compact subsets with non-empty interiors of the finitedimensional real affine spaces $X$ and $X^{\prime}$, respectively, and $W:=$ Anal $(E)$ and $W^{\prime}:=$ $:=\operatorname{Anal}\left(E^{\prime}\right)$ (cf. Examples 6.3), necessary and sufficient conditions are that $\operatorname{dim} X=$ $=\operatorname{dim} X^{\prime}$, that $\sigma$ and $\sigma^{-1}$ be real-analytic in the interior of $E$ and $E^{\prime}$, respectively, and that $h \in$ Anal+ $\left(E^{\prime}\right)$. The proof of the necessity is expedited by the use of the Prineiple of Domain-Invariance (e.g., [4; p. 358]).

If $E$ and $E^{\prime}$ are as in the preceding paragraph, and $W:=\operatorname{Pol}(E)$ and $W^{\prime}:=\mathrm{Pol}\left(E^{\prime}\right)$ it is an amusing and not quite trivial exercise to prove that necessary and sufficient 
conditions are: that $\operatorname{dim} X=\operatorname{dim} X^{\prime}$; that $h$ be a constant; and that $\sigma=\left.\mu\right|_{E^{\prime}} ^{E}$, where $\mu: X^{\prime} \rightarrow X$ is a polynomial mapping that has a polynomial inverse and satisfies $\mu_{>}\left(E^{\prime}\right)=E$. Note that if $\operatorname{dim} X=\operatorname{dim} X^{\prime}=1$ then $\mu$ must be an invertible affine mapping, but that $\mu$ need not be affine if $\operatorname{dim} X=\operatorname{dim} X^{\prime}>1$.

We are interested in cases in which $W:=\operatorname{Aff}(E)$ for a suitable $E$, but which are not immediately treatable by means of Corollaries $8.2,8.3$, or 8.4 ; we therefore require some additional preparation.

Let $X$ be a locally convex Hausdorff topological linear space, and let $E$ be a (nonempty) compact convex subset of $X$. A point $p \in E$ is a semi-exposed point of $E$ in $X$ if for every $q \in E \backslash\{p\}$ there exists $\lambda \in X^{*}$ (the dual space of $X$ ) such that $\sup \lambda_{>}(E)=$ $=\lambda(p)>\lambda(q)$. If one can choose this $\lambda$ independently of $q$, i.e., if there exists $\lambda \in X^{*}$ such that $E \cap \lambda^{>}\left(\left\{\sup \lambda_{>}(E)\right\}\right)=\{p\}$, then $p$ is an exposed point of $E$ in $X$ (ef. [3; p. 105]). Observe that these definitions depend on the topology of $X$.

8.6. THEOREM. - Assume that $E$ is a non-empty compact convex subset of a locally convex Hausdorff topological linear space $X$, and that $E$ is the closed convex hull of the set of its semi-exposed points in $X$. Set $W:=\operatorname{Aff}(E) . A \leqslant-\leqslant-i s o m o r p h i s m p: W^{+} \rightarrow$ $\rightarrow W^{\prime+}$ that is homogeneous at some $w \in W^{+}$is then necessarily a cone-isomorphism.

Proof. - Let $\operatorname{Sexp}(E)$ be the set of semi-exposed points of $E$ in $X$, and let $E_{0}$ be the $X$-closure of Sexp $(E)$, a non-empty compact subset of $E$. Let $W_{0}$ be the subspace of Cont $\left(E_{0}\right)$ consisting of the restrictions to $E_{0}$ of all elements of $A f f(E)$, and let $\varrho: \operatorname{Aff}(E) \rightarrow W_{0}$ be the restriction mapping defined by $\varrho(f):=\left.f\right|_{E_{0}}$ for all $f \in \Delta \mathrm{Af}(E)$; it is of course linear and surjective. We observe that $W_{0}$ satisfies $\left(W_{1}\right)$ and $\left(W_{2}\right)$, since $\operatorname{Aff}(E)$ does.

Since $E$ is the closed convex hull of $\operatorname{Sexp}(E)$ and hence also of $E_{0}$, it follows that, for each $f \in \operatorname{Aff}(E)$, the (closed) convex hull of the compact subset Rng $\varrho(f)=f_{>}\left(E_{0}\right)$ of $\boldsymbol{R}$ is precisely $f_{>}(E)=\operatorname{Rng} f$. In particular, $\varrho(f)=0$ implies $f=0$, so that $\varrho$ is injective, and hence a linear isomorphism. Moreover we have the chain of equivalences

$$
\varrho(f) \in W_{0}^{+} \Leftrightarrow \operatorname{Rng} \varrho(f) \subset \boldsymbol{P}^{\times} \Leftrightarrow \operatorname{Rng} f \subset \boldsymbol{P}^{\times} \Leftrightarrow f \in \operatorname{Aff}^{+}(E)
$$

Therefore $\varrho$ has a restriction to a cone-isomorphism $\psi: \mathrm{Aff}^{+}(E) \rightarrow W_{0}^{+}$.

Let $p \in \operatorname{Sexp}(E)$ be given. For each $q \in E_{0} \backslash\{p\}$ we may choose $\lambda \in X^{*}$ such that $\sup \lambda_{>}(E)=\lambda(p)>\lambda(q)$; we then define $f \in \operatorname{Aff}(E)$ by $f:=\left.(\lambda(p) 1-\lambda)\right|_{E}(1: X \rightarrow \boldsymbol{R}$ is the constant with value 1) and find Rng $\varrho(f) \subset \operatorname{Rng} f=(\lambda(p) 1-\lambda)_{>}(E) \subset P$, so that $\varrho(f) \in \bar{W}_{0}^{+} ;$and also $(\varrho(f))(p)=f(p)=0<\lambda(p)-\lambda(q)=f(q)=(\varrho(f))(q)$. Since $q \in E_{0} \backslash\{p\}$ was arbitrary, we conclude from (3.1) that $p \in P\left(W_{0}\right)$. That $\operatorname{Sexp}(E) \subset P\left(W_{0}\right)$. Since $\operatorname{Sexp}(E)$ is dense in $E_{0}$ by construction, $P\left(W_{0}\right)$ is also dense in $\boldsymbol{E}_{\mathbf{0}}$.

The mapping $\varphi \circ \psi^{-1}: W_{0}^{+} \rightarrow W^{\prime+}$ is a $\leqslant-\leqslant$-isomorphism, and it is homogeneous at $\psi(w) \in W_{0}^{+}$. By Corollary $8.2, \varphi \circ \psi^{-1}$ is a cone-isomorphism; therefore $\varphi=$ $=\left(\varphi \circ \psi^{-1}\right) \circ \psi$ is a cone-isomorphism. 


\section{REFERENCES}

[1] A. D. Aleksandrot - V. V. Ovčinnikova, Notes on the foundations of relativity theory, Vestnik Leningrad Univ., 11 (1953), pp. 95-110 (Russian).

[2] G. Choquet - H. Corson - V. KLeE, Exposed points of convex sets, Pacific J. Math., 17 (1966), pp. 33-43.

[3] M. M. DAX, Normed linear spaces, 3rd ed. (Ergebnisse der Mathematik und ihrer Grenzgebiete, Heft 21), Springer-Verlag, New York - Heidelberg - Berlin (1973).

[4] J. Dugundj, Topology, Allyn and Bacon, Boston (1966).

[5] I. Kaplansky, Lattices of continuous functions, I, Bull. Amer. Math. Soc., 53 (1947), pp. 617-623.

[6] W. NolL - J. J. ScHäFFer, Order isomorphisms in affine spaces, Ann. Mat. Pura Appl., 117 (1978), pp. 243.262.

[7] W. Norx - J. J. SchäfFer, Orders, gauge, and distance in faceless linear cones; with examples relevant to continuum mechanics and relativity, Arch. Rational Mech. Anal., 66 (1977), pp. 345-377.

[8] J. J. SCHÄFFER, Orders, gauge, and distance in faceless linear cones, II: Gauge-preserving bijections are cone-isomorphisms, Arch. Rational Mech. Anal., 67 (1978), pp. 305-313.

[9] I. NAMIOKA - S. SAEKI, On lattice isomorphisms of $O(X)^{+}$(to appear). 